ALEA, Lat. Am. J. Probab. Math. Stat. 18, 35-67 (2021)

DOI: 10.30757/ALEA.v18-03

\title{
Noncommutative analogues of the Law of Small Numbers for random variables indexed by elements of positive symmetric cones
}

\author{
Lahcen Oussi and Janusz Wysoczański \\ Institute of Mathematics \\ University of Wrocław \\ Pl. Grunwaldzki 2/4, \\ 50-384 Wrocław, Poland. \\ E-mail address: lahcen.oussi@math.uni.wroc.pl , jwys@math.uni.wroc.pl
}

\begin{abstract}
We present an analogue of the classical Law of Small Numbers, formulated for the notion of bm-independence, where the random variables are indexed by elements of positive symmetric cones in Euclidean spaces, including $\mathbb{R}_{+}^{d}$, the Lorentz cone in Minkowski spacetime and positive definite real symmetric matrices. The geometry of the cones plays a significant role in the study as well as the combinatorics of bm-ordered partitions.
\end{abstract}

\section{Introduction}

The main objects of study in this paper is the Law of Small Numbers for bmindependent random variables, indexed by partially ordered sets (Wysoczański, 2007, 2008, 2010).

Noncommutative probability grew out of free group properties. Around 1983 Voiculescu $(1985,1986,1995)$ invented free probability based on new notion of independence named free independence (or freeness, for short). The condition for freeness appeared also in the work of Avitzour (1982) on spatial free product of algebras. We recommend Voiculescu et al. (1992) as the basic source of the free probability theory. In 1998 Muraki invented his notion of monotonic independence (Muraki, 2001, 2002), which established a framework for the monotonic probability. Earlier, conditions for the notion of boolean independence appeared in the works of Bożejko (1986), von Waldenfels (1978), Speicher and Woroudi (1997), Ben Ghorbal

Received by the editors November 6th, 2019; accepted October 3rd, 2020.

2010 Mathematics Subject Classification. 46L53, 60F99, 06A06, 05A18.

Key words and phrases. Noncommutative probability, bm-independence, Law of Small Numbers, positive cones, labelled partitions, combinatorics.

Research partially supported by the Polish National Science Center (NCN) grant 2016/21/B/ST1/00628 and by the National Agency for Academic Exchange (NAWA) POLONIUM project PPN/BIL/2018/1/00197/U/00021. 
and Schürmann (2004), allowing a development of the boolean probability. In fact, the construction of boolean independent operators and of the boolean product of representations appeared in Bożejko's paper (Bożejko, 1987) under the name regular free product, where he proved that it preserves uniform boundedness of representations.

Moreover, classifications of the universal notions of independence have been established by Speicher (1997b), Ben Ghorbal and Schürmann (2002), Muraki (2002), Lenczewski (1998), which exhibited that, under some universal conditions, there are only five such notions: the tensor independence (i.e. the classical one), the free independence of Voiculescu, the monotonic and the anti-monotonic independences of Muraki and the boolean independence.

However, outside the universal framework there is a variety of other notions of independence for noncommuting random variables, including mixtures of the above universal ones. In particular, there is conditional freeness introduced by Bożejko et al. (1996) and $q$-independence which appeared in the context of the $q$ deformation defined by Bożejko et al. (1997). The notion of orthogonal independence was introduced by Lenczewski (2007). Młotkowski defined $\Lambda$-boolean independence (Młotkowski, 2004b) and $\Lambda$-free independence (Młotkowski, 2004a), studied also in Speicher and Wysoczański (2016), which are mixtures of classical and boolean/free independences. The mixture of boolean and free independences (bf-independence) was introduced by Kula and the second-named author (Kula and Wysoczański, 2013). Other generalizations of boolean and monotone independences can be found in Hasebe (2011b,a).

The boolean-monotone independence (bm-independence for short) has been introduced by the second-named author (Wysoczański, 2007, 2008, 2010). The idea of this notion was born in the construction of weakly monotonic Fock space, which appeared in Wysoczański (2005) as a model of the monotone independence (for the explanation of the motivation of this construction we refer the reader to the Introduction in Wysoczański (2007), where the influence of Belavkin $(1984,1985)$ is indicated). In fact, it turned out to be a special case of a more general construction of Fock spaces deformed by a Yang-Baxter-Hecke operators, described by Bożejko (2012), which, in turn, was based on the Pusz-Woronowicz construction of twisted commutation relations (Pusz and Woronowicz, 1989).

In general, it was an attempt to generalize Muraki's monotonic independence to random variables indexed by posets. Similar ideas motivated the construction of bf-independence (Kula and Wysoczański, 2013). Recently Jekel and Liu (2020) used the operad framework to show that for any poset one can define independence, which can be expressed in terms of boolean and orthogonal ones.

Several studies were devoted to interpolations between various notions of independence (cf. Bożejko et al., 1997; Lenczewski and Sałapata, 2006; Proskurin and Iksanov, 2003; Młotkowski, 2004b; Franz, 2003; Bożejko et al., 2006; Wojakowski, 2007). Some others have been devoted to deformations of related convolutions, in particular the $t$-deformation introduced in Bożejko and Wysoczański (1998) and studied in Bożejko and Wysoczański (2001); Wojakowski (2007).

As for classical independence, for each notion of noncommutative independence, one defines a convolution of measures as the distribution of the sum of two independent random variables. In this way one obtains convolutions of probability measures 
called free, conditionally free, monotone, boolean, orthogonal and $q$-deformed convolutions. On the other hand, for bm- or bf-independences, two random variables are either boolean independent or free/monotone independent, hence there is no simple way to define related convolution. A procedure to do this is presented in Jekel and Liu (2020), where it is shown that for a tuple of random variables, indexed by a finite poset, one can define the distribution of their sum in terms of the boolean and orthogonal convolutions of their individual distributions.

Within these noncommutative frameworks, especially the one involving freeness, but in part also in the monotonic and boolean cases, analogues of a large part of the classical probability results have been proved, starting from analogues of the Central Limit Theorem (CLT). In the case of freeness it was first shown in Bożejko's study (Bożejko, 1975) of free groups, then by Voiculescu (1985) in the general free setting, and this appeared to be the Wigner semicircle law. A short proof of the free CLT in relation with free Khintchine inequality was also given by Buchholz (1999). The monotonic analogue has been obtained by Muraki (2001) and Lu (1997) and it appeared to be the arcsine law. For the boolean case the analogue of the classical CLT is just the Bernoulli distribution. For the $\Lambda$-free independence and $\Lambda$-boolean independence analogues of classical CLT were obtained by Młotkowski (2004a,b).

Many more classical properties have been studied in the noncommutative context (cf. Arizmendi and Hasebe, 2013; Bożejko et al., 2018; Lenczewski and Sałapata, 2006; Liu, 2018). Moreover, several noncommutative results have been extended to operator valued distributions (cf. Speicher, 1998; Belinschi et al., 2013; Hasebe and Saigo, 2014; Speicher, 1997a; Popa, 2008; Anshelevich and Williams, 2016). More information can be found in Nica and Speicher (2006); Hiai and Petz (2000); Hora and Obata (2007).

On the other hand, more challenging then CLT happend to be the noncommutative analogues of the classical Law of Small Numbers (LSN). This comes from the fact that an additional intensity parameter appears. The generalizations of this limit theorem to noncommutative settings have been obtained by Speicher (1990) for free independence, by Bożejko et al. (1996) for conditionally free independence and by Muraki (2001) for monotone independence. For the study of the related free Poisson processes we refer the reader to Anshelevich (2000).

The basic formulation of the classical Law of Small Numbers can be stated as follows. Consider a sequence $0 \leq \lambda_{N} \leq 1$ with $\lim _{N \rightarrow \infty} \lambda_{N}=\lambda$ and let $\delta_{k}$ be the Dirac measure at $k \in \mathbb{N}$. Then, for the discrete measures $b_{N}:=\left(1-\frac{\lambda_{N}}{N}\right) \delta_{0}+\frac{\lambda_{N}}{N} \delta_{1}$, the limit as $N \rightarrow \infty$ of the $N$-fold (classical) convolution

$$
\underbrace{b_{N} * \cdots * b_{N}}_{\text {N times }}=\sum_{k=0}^{N}\left(\begin{array}{l}
N \\
k
\end{array}\right)\left(1-\frac{\lambda_{N}}{N}\right)^{N-k}\left(\frac{\lambda_{N}}{N}\right)^{k} \delta_{k}
$$

is the Poisson measure $p_{\lambda}:=e^{-\lambda} \sum_{k=0}^{\infty} \frac{\lambda^{k}}{k !} \delta_{k}$ with intensity $\lambda>0$.

The classical, free and monotone Laws of Small Numbers can be formulated more generally as a limit theorem for arrays of random variables. In the noncommutative situation classical independence is replaced by an appropriate noncommutative one.

A noncommutative probability space is a pair $(\mathcal{A}, \varphi)$, where $\mathcal{A}$ is a unital *-algebra and $\varphi$ is a state on $\mathcal{A}$ (i.e. a normalized positive functional $\varphi: \mathcal{A} \rightarrow \mathbb{C}$ ). For noncommutative limit theorems the convergence is considered as the convergence in moments with respect to $\varphi$. 
The reformulation of the classical, free and monotone Laws of Small Numbers is as follows. Assume we are given an array $\left\{X_{j}^{n}: 1 \leq j \leq N\right.$, with $\left.j, N \in \mathbb{N}\right\}$ consisting of (classical or noncommutative) random variables, with the following properties:

(1) for each $N \in \mathbb{N}$, the random variables $\left\{X_{j}^{N}: 1 \leq j \leq N\right\}$ are identically distributed and (classically, freely or monotonically) independent,

(2) there exists $\lambda \in(0,1)$ such that $\lim _{N \rightarrow \infty} N \varphi\left(\left(X_{j}^{N}\right)^{r}\right)=\lambda$, for all $j, r \in \mathbb{N}$.

Then the partial sums $S_{N}:=\sum_{j=1}^{N} X_{j}^{N}$ converge (in moments) to a probability measure, whose moments $\left(m_{r}\right)_{r \geq 0}$ are described by

$$
\lim _{N \rightarrow \infty} \varphi\left[\left(S_{N}\right)^{r}\right]=m_{r}=\sum_{\pi \in \operatorname{Part}(r)} V(\lambda, \pi)
$$

where (cf. Hasebe and Saigo, 2011 Section 6.):

(1) For classical independence, $\operatorname{Part}(r)$ is the set $\mathcal{P}(r)$ of all partitions of $\{1,2, \ldots, r\}$ with $V(\lambda, \pi)=\lambda^{b(\pi)}$ and $b(\pi)$ is the number of blocks of a partition $\pi$,

(2) For free independence, $\operatorname{Part}(r)$ is the set $\mathcal{N} C(r)$ of all non-crossing partitions of $\{1,2, \ldots, r\}$ with $V(\lambda, \pi)=\lambda^{b(\pi)}$,

(3) For monotonic independence, $\operatorname{Part}(r)$ is the set $\mathcal{N} C(r)$ of all non-crossing partitions of $\{1,2, \ldots, r\}$ and $V(\lambda, \pi)$ counts approximately, as $N \rightarrow \infty$, the ratio of the number of all monotonic labellings of blocks of $\pi \in \mathcal{N} C(r)$ with numbers $\{1,2, \ldots, N\}$ by $N^{b(\pi)}$.

For free independence, the limit distribution is called the free Poisson measure and it is the Marchenko-Pastur law, whereas for monotonic independence the monotone Poisson measure has not been identified explicitly, even though formulas for its moments are known. In all these (classical and noncommutative) formulations the random variables are indexed by the totally ordered set of positive integers $\mathbb{N}$. In our study we consider noncommutative random variables which are indexed by a partially ordered set and satisfy the notion of independence called the bm-independence (details are described in the following subsection). The notion of bm-independence combines two universal noncommutative notions of independence: the monotonic independence (Muraki, 2001) and the boolean independence (Bożejko, 1986; Speicher and Woroudi, 1997). Roughly speaking, if the index set has at least two elements, then random variables indexed by incomparable elements are boolean independent, and those indexed by totally ordered elements are monotone independent.

Our main results, the bm-Laws of Small Numbers (bm-LSN for short), will be limit type theorems naturally associated with some partially ordered sets. The formulation of these results requires several introductory explanations. In general, a partial order can be naturally defined on each (real or complex) vector space $\mathcal{X}$ with a positive cone $\Pi \subset \mathcal{X}$ (i.e. a subset closed under sums of vectors and multiplication by positive reals). Given such positive cone, one defines the partial order $\preceq$ on $\mathcal{X}$ by putting $u \preceq v$ if $v-u \in \Pi$, for $u, v \in \mathcal{X}$. Recall, that for a poset $(\mathcal{X}, \preceq)$ and two elements $\xi \preceq \eta \in \mathcal{X}$ one defines interval $[\xi, \eta]:=\{\rho \in \mathcal{X}: \xi \preceq \rho \preceq \eta\}$ (with the use of strict relation $\prec$ for open/half open intervals). The specific feature of a partial order is that some elements $u, v \in \mathcal{X}$ are incomparable, i.e. neither $u \preceq v$ nor $v \preceq u$ 
(which we will denote by writing $u \nsim v$ ). We will be interested in the following partial orders, which are defined by positive cones $\Pi$ in Euclidean spaces $\mathcal{X}$ :

(1) $\Pi=\mathbb{R}_{+}^{d}$ in $\mathcal{X}=\mathbb{R}^{d}$; then $\left(a_{a}, \ldots, a_{d}\right) \preceq\left(b_{1}, \ldots, b_{d}\right)$ if $a_{j} \leq b_{j}$ for all $1 \leq j \leq d$.

(2) The Lorentz light cones $\Pi=\Lambda_{d}^{1}$ in $(d+1)$-dimensional Minkowski's spacetime $\mathcal{X}=\mathbb{R}_{+} \times \mathbb{R}^{d}$. In this case $\xi:=(t ; x) \in \Lambda_{d}^{1}$ if $t \in \mathbb{R}_{+}, x=\left(x_{1}, \ldots, x_{d}\right) \in$ $\mathbb{R}^{d}$ and $t>\|x\|=\sqrt{x_{1}^{2}+\cdots+x_{d}^{2}}$; moreover, the partial order is given by $(t ; x) \preceq(s ; y)$ if $s-t>\|y-x\|$, i.e. $(s ; y)$ lies in the future cone of $(t ; x)$.

(3) $\Pi=\operatorname{Symm}_{d}^{+}(\mathbb{R})$ is the cone of real symmetric positive definite matrices in $\mathcal{X}=\mathbb{M}_{d}(\mathbb{R}) ;$ then the partial order is defined as $\xi \preceq \rho$ if $\rho-\xi \in \operatorname{Symm}_{d}^{+}(\mathbb{R})$ for $\xi, \rho \in \mathbb{M}_{d}(\mathbb{R})$.

In this paper we will consider only these three types of positive cones. They form an important subclass of the positive symmetric cones (for the classification and properties of which we refer to Faraut and Korányi, 1994). These positive symmetric cones have nice geometric structure and can be embedded in $\mathbb{R}^{m}$, for some (minimal) $m \in \mathbb{N}$. In particular, there are formulas for the volumes of intervals, which are crucial in our bm-LSN. Our proof uses also the volume characteristics of these positive symmetric cones, the notion introduced in Kula and Wysoczański (2010).

Theorem 1.1 (Volume characteristic, Kula and Wysoczański, 2010). For each of the aforementioned positive symmetric cones $\Pi$ there exists a sequence $\left(\gamma_{n}(\Pi)\right)_{n \geq 1}$ such that for any $\xi \in \Pi$ and any $n \in \mathbb{N}$

$$
\gamma_{n}(\Pi)=\frac{1}{v(\xi)^{n}} \int_{\rho \in[0, \xi]} v(\rho)^{n-1} d(\rho),
$$

where $v(\xi)$ denotes the Euclidean volume of the interval $[0, \xi] \subset \Pi \subset \mathbb{R}^{m}$ and $d(\rho)$ is the Lebesgue measure on $\mathbb{R}^{m}$ (the dimension $m$ is minimal for the embedding $\left.\Pi \subset \mathbb{R}^{m}\right)$.

Thus, the sequence $\left(\gamma_{n}(\Pi)\right)_{n \geq 0}$ constitutes a geometric invariant of the cone $\Pi$. In what follows we will simply write $\gamma_{n}$ for $\gamma_{n}(\Pi)$ if the positive cone $\Pi$ is fixed.

Remark 1.2. The volume characteristic sequence appears as the generalization of the following property of the positive real half-line:

$$
\frac{1}{t^{n}} \int_{0}^{t} s^{n-1} d s=\frac{1}{n}=\gamma_{n}\left(\mathbb{R}_{+}\right)
$$

as $v([0, s])=s$ for $s>0$.

Example 1.3. For the reader's convenience we present some of these volume characteristic sequences (see Kula and Wysoczański, 2010 Corollary 4), of which we will make use in the presentation of Examples 4.2

(1) For $\Pi=\mathbb{R}^{d}$ we have $\gamma_{n}=n^{-d}$.

(2) For $\Pi=\Lambda_{1}^{1}$ we have $\gamma_{n}=n^{-2}$.

(3) For $\Pi=\Lambda_{2}^{1}$ and $\Pi=\operatorname{Symm}_{2}^{+}(\mathbb{R})$ we have $\gamma_{n}=\frac{24}{3 n(3 n-1)(3 n+1)}$.

(4) For $\Pi=\Lambda_{3}^{1}$ we have $\gamma_{n}=\frac{6}{2 n^{2}(2 n-1)(2 n+1)}$.

Several modifications have to be made to the statement of the law of small numbers when we consider random variables indexed by a partially ordered set. As 
we replace the real numbers $\mathbb{R}$ by a partially ordered set $(\mathcal{X}, \preceq)$, the positive reals $\mathbb{R}_{+}$are replaced by a positive cone $\Pi \subset \mathcal{X}$.

The first problem is the range of summation for the partial sums, and this is solved by considering intervals. Namely, the partial sum $S_{N}=\sum_{j=0}^{N} X_{j}^{N}$ can be rewritten as $\sum_{j \in[0, N] \cap \mathbb{N}} X_{j}^{N}$, which shows that the summation is over a finite discrete subset $[0, N] \cap \mathbb{N}$ of an interval $[0, N] \subset \mathbb{R}_{+}$in the cone of positive reals. In general, an interval $[0, \xi] \subset \Pi$ is infinite (uncountable, like a real interval $\left.[0, N] \subset \mathbb{R}_{+}\right)$, so it is necessary to find a proper discrete subset $\mathbf{I} \subset \Pi$ in the positive cone, which would play the role of positive integers $\mathbb{N} \subset \mathbb{R}_{+}$and make the intervals $[0, \xi] \cap \mathbf{I}$ finite, for all $\xi \in \Pi$.

The second problem is to define $\mathbf{I} \subset \Pi$ such that the cardinality of the finite intersection $|[0, \xi] \cap \mathbf{I}|$ approximates the volume $v(\xi)$ of the interval $[0, \xi] \in \Pi$. For this purpose we give the following definition.

Definition 1.4. Let $\mathbf{I}$ denote the following discrete subsets in the positive symmetric cones:

(1) $\mathbf{I}:=\mathbb{N}^{d}$ if $\Pi=\mathbb{R}_{+}^{d}$,

(2) $\mathbf{I}:=\mathbb{N} \times \mathbb{Z}^{d}$ if $\Pi=\Lambda_{d}^{1}$ is the Lorentz cone,

(3) $\mathbf{I}:=\operatorname{Symm}_{d}^{+}(\mathbb{Z})$ if $\Pi=\operatorname{Symm}_{d}^{+}(\mathbb{R}) \subset \mathbb{M}_{d}(\mathbb{R})$.

In particular, the set $\mathbf{I}$ has a finite intersection with any interval $[\rho, \eta] \subset \Pi$ and for this intersection we will use the notation $[\rho, \eta]_{\mathbf{I}}:=[\rho, \eta] \cap \mathbf{I}$. Therefore, eventually the summation $\sum_{j=0}^{N} X_{j}^{N}=\sum_{j \in[0, N] \cap \mathbb{N}} X_{j}^{N}$ is replaced by the finite summation $\sum_{\rho \in[0, \xi] \mathrm{I}} X_{\rho}^{\xi}$.

The third problem is to give a proper meaning to the convergence $\xi \rightarrow \infty$ for $\xi \in \mathcal{X}$ and this requires an additional structure on $\mathcal{X}$. In the classical case, $N \rightarrow \infty$ is equivalent to $v(N)=\operatorname{vol}([0, N]) \rightarrow \infty$, so the natural choice would be that $v(\xi) \rightarrow \infty$. This is necessary but not sufficient for our method of proof. That is why we define the following.

Definition 1.5. For $\xi \in \Pi$, we define the convergence $\xi \stackrel{\Pi}{\rightarrow} \infty$ depending on the positive cone under consideration:

(1) if $\xi:=\left(a_{1}, \ldots, a_{d}\right) \in \Pi=\mathbb{R}_{+}^{d}$, then $\xi \stackrel{\Pi}{\rightarrow} \infty$ if $a_{j} \rightarrow \infty$ for all $1 \leq j \leq d$,

(2) if $\xi:=(t ; x) \in \Pi=\mathbb{R}_{+} \times \mathbb{R}^{d}=\Lambda_{d}^{1}$ is in the respective Lorentz cone, then $\xi \stackrel{\Pi}{\rightarrow} \infty$ if $t-\|x\| \rightarrow \infty$

(3) if $\xi \in \Pi=\operatorname{Symm}_{d}^{+}(\mathbb{R}) \subset \mathbb{M}_{d}(\mathbb{R})$ and $0<\lambda_{1} \leq \cdots \leq \lambda_{d}$ are the eigenvalues of $\xi$, then $\xi \stackrel{\Pi}{\rightarrow} \infty$ if $\lambda_{1} \rightarrow \infty$.

Remark 1.6. The conditions for $\xi \stackrel{\Pi}{\rightarrow} \infty$ guarantee that $v(\xi):=\operatorname{vol}[0, \xi] \rightarrow \infty$ as $\xi \stackrel{\Pi}{\rightarrow} \infty$. This follows from the following formulas for the volumes of intervals $[0, \xi] \subset \Pi$ (c.f. Faraut and Korányi, 1994):

(1) $v(\xi)=\prod_{j=1}^{d} a_{j}$ if $\xi:=\left(a_{1}, \ldots, a_{d}\right) \in \Pi=\mathbb{R}_{+}^{d}$,

(2) $v(\xi)=\alpha_{d}\left(t^{2}-\|x\|^{2}\right)^{\frac{d+1}{2}}$ for some constant $\alpha_{d}$, if $\xi:=(t ; x) \in \Pi=\Lambda_{d}^{1}$, 
(3) $v(\xi)=\beta_{d}\left(\prod_{j=1}^{d} \lambda_{j}\right)^{\frac{d+1}{2}}=\beta_{d}[\operatorname{det}(\xi)]^{\frac{d+1}{2}}$ for some constant $\beta_{d}$, if $\left(\lambda_{1}, \ldots, \lambda_{d}\right)$ are the eigenvalues of $\xi \in \Pi=\operatorname{Symm}_{d}^{+}(\mathbb{R}) \subset \mathbb{M}_{d}(\mathbb{R})$.

Remark 1.7. On the other hand, the condition $v(\xi) \rightarrow \infty$ is not equivalent to $\xi \stackrel{\Pi}{\rightarrow} \infty$, as one can see by taking $\xi_{n}=\left(n^{2}, \frac{1}{n}\right) \in \mathbb{R}_{+}^{2}$. Then $v\left(\xi_{n}\right)=n \rightarrow \infty$, but not $\xi \stackrel{\Pi}{\rightarrow} \infty$.

We will also need the following definition of a limit of a function $f(\xi)$ as $\xi \stackrel{\Pi}{\rightarrow} \infty$.

Definition 1.8. For a function $f: \Pi \mapsto \mathbb{R}$ we will write $\lim f(\xi)=\alpha$ if for each $\varepsilon>0$ there exists $\mu \in \Pi$ such that for every $\xi \in \Pi$ if $\mu \preceq \xi$ then $|f(\xi)-\alpha|<\varepsilon$.

The fourth (more technical) problem is the normalization factor: in the above formulation of the bm-LSN, the factor $N$ in the condition $\lim _{N \rightarrow \infty} N \cdot \varphi\left(\left(X_{j}^{N}\right)^{r}\right)=\lambda$ must be replaced by one suitable for considered positive symmetric cones. One nat-

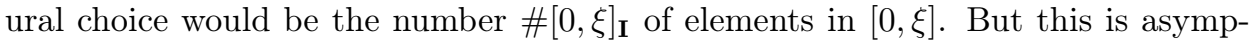
totically the same as the volume $v(\xi)$, and thus we will write the condition as $\lim _{\bar{\Pi}} v(\xi) \cdot \varphi\left(\left(X_{\rho}^{\xi}\right)^{r}\right)=\lambda$ (for each $\rho \in[0, \xi]_{\mathbf{I}}$ and each $r \in \mathbb{N}$ ). However, it turns out $\xi \stackrel{\Pi}{\rightarrow} \infty$

that in fact we will need this convergence to be controlled uniformly on intervals $[0, \xi]$.

The fifth change is that we consider the (noncommutative) notion of $\mathrm{bm}$ independence instead of the classical one. It differs from other noncommutative independences in that it concerns random variables indexed by arbitrary partially ordered set instead of the positive integers.

The paper is organized as follows.

In Section 2, we introduce basic notions of noncommutative probability, give the definition of bm-independence and provide preliminary formulation (Theorem 2.4) of our main result. We also describe an algorithm for computing joint moments using bm-independence.

Section 3 contains information about bm-ordered non-crossing partitions and provides combinatorial tools (especially Corollary 3.13) for proving our main results. In particular, in computation of joint moments we use properties of naturally related partitions (Definition 3.2). In Lemma 3.11 we construct a refinement of partition related with a given moment, and show its properties. In Theorem 3.12 we describe details of the algorithm which produces the coarsest non-crossing strictly bm-ordered partition, which is a refinement of the one given in Definition 3.2. Moreover, we show that the two refinements are in fact equal.

In Section 4, we give the detailed formulation (Theorem 4.4) of the main theorem. We also give examples of moments of the limiting measures in the classical, free and monotone cases as well as two of our results.

Sections 5 and 6 contain the proof of our main result for each class of the considered positive cones. In particular, in Section 5 we show a combinatorial reduction of computation of limits of joint moments, using the algorithm from Section 3. Then we further reduce the proof to estimating the cardinality of bm-orders on non-crossing partitions, with labels taken from (a finite subset of a) given interval. In particular, in Section 6 we show that for each positive cone under consideration 
the ratio of this cardinality by (an appropriate power of) the volume of the interval has a limit, which depends on the cone and the partition only.

In Section 7, we mention some open problems related to this study.

\section{Preliminaries}

In this section we introduce basic notions and preliminary formulations of our main result.

2.1. Basic notions of noncommutative probability. A noncommutative probability space is a pair $(\mathcal{A}, \varphi)$ of a unital *-algebra and a state $\varphi$ on it. Recall that a state on $\mathcal{A}$ is a linear functional $\varphi: \mathcal{A} \rightarrow \mathbb{C}$ such that $\varphi\left(a^{*} a\right) \geq 0$ for all $a \in \mathcal{A}$ and $\varphi\left(1_{\mathcal{A}}\right)=1$ for the unit element $1_{\mathcal{A}} \in \mathcal{A}$. A noncommutative random variable is a self-adjoint element $a=a^{*} \in \mathcal{A}$. In noncommutative probability the state plays a similar role as the expectation in classical probability (actually they coincide if $\mathcal{A}$ is commutative). In particular, one defines the distribution $\mu_{a}$ of a random variable $a=a^{*} \in \mathcal{A}$ (with respect to $\varphi$ ) as the probability measure $\mu_{a}$ for which $\left(\varphi\left(a^{n}\right)\right)_{n \geq 0}$ is the moment sequence:

$$
\varphi\left(a^{n}\right)=\int_{-\infty}^{+\infty} t^{n} \mu_{a}(d t)
$$

The existence of a distribution $\mu_{a}$ of a random variable $a \in \mathcal{A}$ is guaranteed by the solution of the Hamburger Moment Problem, since the sequence $\left(\varphi\left(a^{n}\right)\right)_{n \geq 0}$ is positive definite, i.e. $\sum_{m, k=0}^{N} \varphi\left(a^{m+k}\right) \lambda_{m} \overline{\lambda_{k}} \geq 0$ for all $N \in \mathbb{N}$ and all $\lambda_{1}, \ldots, \lambda_{N} \in$ $\mathbb{C}$. The uniqueness of $\mu_{a}$ follows if, for instance, the moments do not growth too fast. This is the case if one considers $\mathcal{A}$ to be a $C^{*}$-algebra and $a=a^{*} \in \mathcal{A}$ an element with finite norm $\|a\|$; since then the moments growth is bounded by the geometric progression $\left(\|a\|^{n}\right)_{n \geq 0}$ and the measure $\mu_{a}$ has compact support included in the interval $[-\|a\|,\|a\|]$.

2.2. Partial orders and bm-independence. Recall that a set $\mathcal{X}$ is partially ordered by a relation $\preceq$ if the relation is

(1) reflexive, i.e. $\xi \preceq \xi$ for $\xi \in \mathcal{X}$,

(2) antisymmetric, i.e. $\xi \preceq \eta$ and $\eta \preceq \xi$ then $\xi=\eta$ for $\xi, \eta \in \mathcal{X}$,

(3) transitive, i.e. $\xi \preceq \eta$ and $\eta \preceq \rho$ then $\xi \preceq \rho$ for $\xi, \eta, \rho \in \mathcal{X}$.

If $(\mathcal{X}, \preceq)$ is partially ordered, then for $\xi, \eta \in \mathcal{X}$, we will write $\xi \prec \eta$ if $\xi \preceq \eta$ and $\xi \neq \eta$; we will also write $\xi \sim \eta$ if $\xi$ and $\eta$ are comparable (i.e. either $\xi \preceq \eta$ or $\eta \preceq \xi$ ) and $\xi \nsim \eta$ if $\xi$ and $\eta$ are incomparable.

Now we recall the notion of bm-independence, which was defined in Wysoczański (2010, Definition 2.1).

Definition 2.1 (bm-independence). Let $(\mathcal{A}, \varphi)$ be a noncommutative probability space defined in 2.1. We say that a family $\left\{\mathcal{A}_{\eta}: \eta \in \mathcal{X}\right\}$ of subalgebras of $\mathcal{A}$, indexed by a partially ordered set $(\mathcal{X}, \preceq)$, is bm-independent in $(\mathcal{A}, \varphi)$ if the following two conditions hold:

BM1: If $\xi, \eta, \rho \in \mathcal{X}$ satisfy: $\xi \prec \rho \succ \eta$ or $\xi \nsim \rho \succ \eta$ or $\xi \prec \rho \nsim \eta$, then for any $a_{1} \in \mathcal{A}_{\xi}, a_{2} \in A_{\rho}, a_{3} \in \mathcal{A}_{\eta}$ we have

$$
a_{1} a_{2} a_{3}=\varphi\left(a_{2}\right) \cdot a_{1} a_{3} \text {. }
$$


BM2: If $\xi_{1} \succ \cdots \succ \xi_{m} \nsim \cdots \nsim \xi_{k} \prec \cdots \prec \xi_{n}$ for some $1 \leq m \leq k \leq n$ and $\xi_{1}, \ldots, \xi_{n} \in \mathcal{X}$, with $a_{j} \in \mathcal{A}_{\xi_{j}}$ for $1 \leq j \leq n$, then

$$
\varphi\left(a_{1} \ldots a_{n}\right)=\prod_{j=1}^{n} \varphi\left(a_{j}\right)
$$

Noncommutative random variables $\left\{a_{\xi} \in \mathcal{A}: \xi \in \mathcal{X}\right\}$ are called bm-independent if the subalgebras $\mathcal{A}_{\xi}$ they generate are bm-independent. Recall that in this definition one gets the monotone independence if $\mathcal{X}$ is totally ordered, and the boolean independence if none of the elements of $\mathcal{X}$ are comparable (Kula and Wysoczański, 2010; Wysoczański, 2010).

Remark 2.2. The reason for calling the conditions [BM1], [BM2] independence is that these conditions allow to compute all joint moments $\varphi\left(a_{1} \ldots a_{n}\right)$ of bmindependent random variables $a_{1}, \ldots, a_{n}$ via marginals $\varphi_{j}:=\left.\varphi\right|_{\mathcal{A}_{j}}$ i.e. by the restrictions of $\varphi$ to the subalgebras they generate (Wysoczański, 2010, Lemmas 2.3, 2.4).

Remark 2.3. The algorithm for evaluating joint moments using the rules [BM1], [BM2] can be described in a purely combinatorial way as follows.

Suppose that $\left\{\mathcal{A}_{\xi}: \xi \in \mathcal{X}\right\}$ are bm-independent in $(\mathcal{A}, \varphi)$ and we want to evaluate joint moment $\varphi\left(a_{1} a_{2} \ldots a_{n}\right)$ where $a_{j} \in \mathcal{A}_{\xi_{j}}$. By applying [BM1], [BM2], this is evaluated as a product of certain moments, each of which only involve terms from the same algebra $\mathcal{A}_{\xi}$. The elements $\xi_{1}, \xi_{2}, \ldots, \xi_{n}$ give rise to a labelled partition $\pi$ of $[n]:=\{1,2, \ldots, n\}$ (see Definition 3.4 and comments after it), where each block $B$ is the set of all elements $j$ where $\xi_{j}$ is equal to some $\xi$, and the label of this block is $l(B)=\xi$. The algorithm shows that there exists a non-crossing partition $\tau=\left(B_{1}, \ldots, B_{k}\right)$ of $[n]$ that refines $\pi$ such that

$$
\varphi\left(a_{1} a_{2} \ldots a_{n}\right)=\prod_{i=1}^{k} \varphi\left(\prod_{j \in B_{i}} a_{j}\right)
$$

where (for each $i$ ) the terms $\left(a_{j}\right)_{j \in B_{i}}$ are multiplied from left to right in increasing order of indices.

[BM1] tells us that if $B$ is an interval block of $\pi$ that with $l(B)$ satisfies the right conditions with respect to the adjacent indices ${ }^{1}$, then $B$ will be a block of $\tau$ and $\tau \backslash B$ can be computed by applying the algorithm to $\tau \backslash B$ recursively.

[BM2] tells us that if $\xi_{1} \succ \cdots \succ \xi_{k} \nsim \cdots \nsim \xi_{l} \prec \cdots \prec \xi_{n}$, then $\tau$ is the trivial (finest) partition into singletons. More generally, if the indices $\xi_{j}$ are as above and if $a_{j}$ is replaced by a string of several elements from $\mathcal{A}_{\xi_{j}}$, then $\tau$ would be an interval partition with one interval block for each $j \in[n]$.

We will show more properties of this algorithm in Theorem 3.12.

2.3. Preliminary formulation of the main result. Now we give a "draft" statement of the bm-LSN. A more precise formulation will be given in Theorem 4.4 after we introduce some more combinatorial objects. To emphasize the analogy with classical probability theory, we use the letter $X$ for our random variables.

\footnotetext{
${ }^{1} B=(p, p+1, \ldots, p+s)$ and $\xi=\xi_{p-1}, \rho=l(B), \eta=\xi_{p+s+1}$ satisfy the assumption in [BM1]: $\xi \prec \rho \succ \eta$ or $\xi \nsim \rho \succ \eta$ or $\xi \prec \rho \nsim \eta$
} 
Theorem 2.4 (bm-LSN, draft). Let $\Pi=\mathbb{R}_{+}^{d}, \Pi=\Lambda_{d}^{1}$ or $\Pi=\operatorname{Symm}_{d}^{+}(\mathbb{R})$ and let $\mathbf{I} \subset \Pi$ be the respective discrete subset. Let $(\mathcal{A}, \varphi)$ be a noncommutative probability space and let $\left\{X_{\rho}^{\xi} \in \mathcal{A}: \rho, \xi \in \mathbf{I}, 0 \preceq \rho \preceq \xi\right\}$ be an array of random variables. Assume that

(1) for each $\xi \in \mathbf{I}$, the random variables $\left\{X_{\rho}^{\xi} \in \mathcal{A}: 0 \preceq \rho \preceq \xi, \rho \in \mathbf{I}\right\}$ are bm-independent in $(\mathcal{A}, \varphi)$;

(2) there exists a constant $\lambda>0$ such that for all $\rho \in \mathbf{I}$ and for all $n \in \mathbb{N}$

$$
\lim _{\xi \rightarrow \infty} \sup _{\rho \in[0, \xi]_{\mathbf{I}}}\left|v(\xi) \cdot \varphi\left(\left(X_{\rho}^{\xi}\right)^{n}\right)-\lambda\right|=0 .
$$

For $\xi \in \mathbf{I}$, define the partial sum $S_{\xi}:=\sum_{\rho \in[0, \xi]_{\mathbf{I}}} X_{\rho}^{\xi}$, then for every $n \in \mathbb{N}$ there exists some combinatorial function $V(\pi)$ defined on the set $\mathcal{N C}(n)$ of all non-crossing partitions such that

$$
\lim _{\xi \rightarrow \infty} \varphi\left(\left(S_{\xi}\right)^{n}\right)=\sum_{\pi \in \mathcal{N C}(n)} \lambda^{b(\pi)} V(\pi) .
$$

Remark 2.5. The assumption (1) does not require that the random variables are identically distributed. The assumption (2) is stronger than in classical, free or monotonic formulations, since it requires convergence to 0 , as $\xi \stackrel{\Pi}{\rightarrow} \infty$, of the suprema of the functions $f_{n}(\xi, \rho):=\left|v(\xi) \cdot \varphi\left(\left(X_{\rho}^{\xi}\right)^{n}\right)-\lambda\right|$, for each $\xi \in \mathbf{I}$, taken over all $\rho \in[0, \xi]_{\mathbf{I}}$ and independent of $n \in \mathbb{N}$.

Remark 2.6. The meaning of the notation $\mathcal{N C}(n)$ and $b(\pi)$ for $\pi \in \mathcal{N C}(n)$ will be explained in the next section.

\section{Partitions and bm-orders}

In this section, we present basic combinatorial objects related to the notion of bm-independence. In particular we describe properties of non-crossing partitions with blocks labeled by elements of a partially ordered $\operatorname{set}(\mathcal{X}, \preceq)$.

First we define partitions of a set. Let $(J, \preceq)$ be a totally ordered finite set. Let $k \in \mathbb{N}$ and assume that $B_{1}, \ldots, B_{k} \subset J$ are pairwise disjoint subsets: $B_{j} \cap B_{i}=$ $\emptyset$ for all pairs of different numbers $1 \leq i \neq j \leq k$, and that their union is $J$ : $B_{1} \cup \cdots \cup B_{k}=J$. Then the set $\pi:=\left\{B_{1}, \ldots, B_{k}\right\}$ is called a partition of $J$ and each $B_{j} \in \pi(1 \leq j \leq k)$ is called a block of the partition $\pi$. For a subset $I \subseteq J$ we denote by $|I|$ the number of elements in $I$. By $b(\pi)=k$ we denote the number of blocks of $\pi$, by $\mathcal{P}(J)$ we denote the set of all partitions of $J$, including, in particular, the full partition $\pi_{J}:=\{J\}$. Moreover, if $|J|=n$ and $1 \leq k \leq n$, then $\mathcal{P}(J ; k)$ will denote the collection of all partitions in $\mathcal{P}(J)$ with $k$ blocks, i.e. $b(\pi)=k$.

Since $J$ is totally ordered, is makes sense to define the minimal element $\min B_{j} \in$ $B_{j}$ and the maximal element $\max B_{j} \in B_{j}$ of the block $B_{j} \in \pi$.

If $\min B_{j}=\max B_{j}$, then the block $B_{j}$ consists of this one element $B_{j}=$ $\left\{\max B_{j}\right\}$ and is called a singleton.

If a block $B_{j} \in \pi$ is of the form $B_{j}:=\left\{a \in J: \min B_{j} \preceq a \preceq \max B_{j}\right\}$, then we call $B_{j}$ an interval block.

If there are two blocks $B_{j} \neq B_{i} \in \pi$ and elements $a_{1}, a_{2} \in B_{i}, b_{1}, b_{2} \in B_{j}$, for which $a_{1} \prec b_{1} \prec a_{2} \prec b_{2}$, then we say that the partition $\pi \in \mathcal{P}(J)$ has a crossing. 
If a partition $\pi \in \mathcal{P}(J)$ does not have crossings, then we call $\pi$ a non-crossing partition; the set of all non-crossing partitions in $\mathcal{P}(J)$ will be denoted by $\mathcal{N C}(J)$.

Definition 3.1. If $1 \leq k \leq n$, then $\mathcal{P}(J ; k) \subset \mathcal{P}(J)$ denotes the set of all partitions $\pi \in \mathcal{P}(J)$ with $b(\pi)=k$ blocks. By $\mathcal{N C}(J ; k) \subset \mathcal{N C}(J)$ we denote the set of all non-crossing partitions $\pi \in \mathcal{N C}(J)$ with $b(\pi)=k$ blocks.

For a nonempty subset $I \subset J$ we consider the restriction $\pi^{\prime}:=\pi \uparrow_{I}$ of $\pi$ to $I$, which is defined as $\pi^{\prime}:=\left\{B_{1}^{\prime}, \ldots, B_{k}^{\prime}\right\}$ with $B_{j}^{\prime}:=B_{j} \cap I$.

We define a partial order on the blocks of a non-crossing partition as follows. For a non-crossing partition $\pi=\left\{B_{1}, \ldots, B_{k}\right\} \in \mathcal{N C}(J)$ we will write

$$
B_{i} \preceq_{\pi} B_{j} \quad \text { if } \quad \min B_{i} \preceq \min B_{j} \preceq \max B_{j} \preceq \max B_{i} .
$$

Since the blocks are disjoint, we have $\min B_{i}=\min B_{j}$ if and only if $\max B_{j}=$ $\max B_{i}$ if and only if $B_{i}=B_{j}$. Hence, if $B_{i} \neq B_{j}$ then (3.1) becomes: $\min B_{i} \prec$ $\min B_{j} \preceq \max B_{j} \prec \max B_{i}$. In such case we will write $B_{i} \prec_{\pi} B_{j}$ and say that the block $B_{j}$ is inside the block $B_{i}$ (equivalently $B_{i}$ is outside $B_{j}$ ). In particular, we will call a block $B_{j} \in \pi$ inner if there exists $1 \leq i \leq b(\pi)$ such that $B_{i} \prec_{\pi} B_{j}$. On the other hand, a block which is not inside any other block will be called outer. The relation $\preceq_{\pi}$ is a partial order on the blocks and the chains of it (i.e. the totally ordered sequences of blocks) are well defined; in particular a block which is not outside any other is contained in a maximal chain.

For $J=[n]:=\{1,2, \ldots, n\}$ we use the notation $\mathcal{P}(n)$ for the set of all partitions of $[n]$ and $\mathcal{N C}(n)$ for the collection of all non-crossing partitions of $[n]$.

If $\left(\rho_{1}, \ldots, \rho_{n}\right)$ is a sequence of elements of $\mathcal{X}$, then there is a natural partition $\pi \in \mathcal{P}(n)$ associated in the following way.

Definition 3.2. Let $\left(\rho_{1}, \ldots, \rho_{n}\right)$ be a sequence of elements of $\mathcal{X}$. For $1 \leq k \leq n$, define $B(k):=\left\{1 \leq j \leq n: \rho_{j}=\rho_{k}\right\}$, then $B(k) \subset[n]$ and for $i \neq j$ we have either $B(i)=B(j)$ or $B(i) \cap B(j)=\emptyset$. In fact, the relation " $i \sim j$ provided $\rho_{i}=\rho_{j}$ " is an equivalence relation on $[n]$ and the blocks are the equivalence classes. In this fashion we obtain a partition $\pi:=\left\{B_{1}, \ldots, B_{k}\right\} \in \mathcal{P}(n)$, where the subsets $B_{1}, \ldots, B_{k}$ are disjoint, their union is $[n]$, and for each $1 \leq j \leq k$ we have $B_{j}=B(i)$ for some $1 \leq i \leq n$. We will say that the partition $\pi$ is associated with sequence $\left(\rho_{1}, \ldots, \rho_{n}\right)$ and denote this by $\pi \sim\left(\rho_{1}, \ldots, \rho_{n}\right)$ or $\left(\rho_{1}, \ldots, \rho_{n}\right) \sim \pi$.

Example 3.3. For $n=8$ let $\left(\rho_{1}, \ldots, \rho_{8}\right)$ be a sequence of elements of $\mathcal{X}$ such that $\xi_{1}:=\rho_{1}=\rho_{3}=\rho_{6}, \xi_{2}:=\rho_{2}=\rho_{5}=\rho_{8}$ and $\xi_{3}:=\rho_{4}=\rho_{7}$, then we obtain the partition $\pi=\left\{B_{1}, B_{2}, B_{3}\right\} \in \mathcal{P}(8)$ where $B_{1}=B\left(\xi_{1}\right)=\{1,3,6\}, B_{2}=B\left(\xi_{2}\right)=\{2,5,8\}$ and $B_{3}=B\left(\xi_{3}\right)=\{4,7\}$

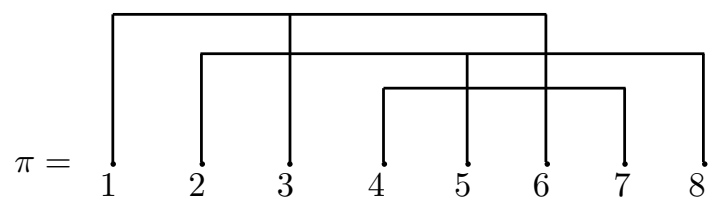

Observe that for a given sequence the associated partition $\pi$ is unique. Moreover, since the blocks of the partition $\pi$ connect equal elements of the associated sequence, it is natural to consider the blocks of $\pi$ as labeled by these elements. 
Definition 3.4. Let $\pi \sim\left(\rho_{1}, \ldots, \rho_{n}\right)$ and for $\rho \in\left\{\rho_{1}, \ldots, \rho_{n}\right\}$ let $B(\rho) \in \pi \in \mathcal{P}(n)$ be the block considered in Definition 3.2. Then $l(B(\rho)):=\rho$ will be called the label of the block $B(\rho)$.

If a partition $\pi:=\left\{B_{1}, \ldots, B_{k}\right\} \in \mathcal{P}(n)$ is associated with a given sequence $\left(\rho_{1}, \ldots, \rho_{n}\right)$, then the sets $\left\{\rho_{1}, \ldots, \rho_{n}\right\}$ and $\left\{l\left(B_{1}\right), \ldots, l\left(B_{k}\right)\right\}$ are equal. In such a case we will call $\left\{l\left(B_{1}\right), \ldots, l\left(B_{k}\right)\right\}$ the label set of the partition $\pi \sim\left(\rho_{1}, \ldots, \rho_{n}\right)$, so the sequence $\left(\rho_{1}, \ldots, \rho_{n}\right)$ gives rise to a labelled partition $\pi$. In the following definitions, we establish an order on this set of labels which will produce a sequence of labels associated in a unique way with such a partition.

Definition 3.5. For a partition $\pi \in \mathcal{P}(n)$ with $b(\pi)=k$ blocks, we will order the blocks according to their minimal elements. By this we mean that $\left(B_{1}, \ldots, B_{k}\right)$ are ordered blocks of $\pi$ if the function $\{1,2, \ldots, k\} \ni j \mapsto \min B_{j}$ is strictly increasing.

Definition 3.6. If $\pi=\left\{B_{1}, \ldots, B_{k}\right\}$ is associated to a sequence $\left(\rho_{1}, \ldots, \rho_{n}\right)$ of elements of $\mathcal{X}$ and $l\left(B_{1}\right), \ldots, l\left(B_{k}\right) \in \mathcal{X}$ are (pairwise different) labels of the blocks, then the sequence $\left(l\left(B_{1}\right), \ldots, l\left(B_{k}\right)\right)$ is uniquely defined and will be called the label sequence of the partition $\pi$; in such a case we will write $\left(l\left(B_{1}\right), \ldots, l\left(B_{k}\right)\right) \approx \pi$ or $\pi \approx\left(l\left(B_{1}\right), \ldots, l\left(B_{k}\right)\right)$.

Remark 3.7. Formally, a partition is a collection $\pi=\left\{B_{1}, \ldots, B_{k}\right\} \in \mathcal{P}(n)$ of disjoint sets, whose union is $[n]$. We will also write $\pi$ as a tuple $\left(B_{1}, \ldots, B_{k}\right)$, where the blocks are ordered in the increasing way as defined above. In particular, we always have $1 \in B_{1}$ and if $\pi \sim\left(\rho_{1}, \ldots, \rho_{n}\right)$, then $B\left(\rho_{1}\right)=B_{1}$ and $l\left(B_{1}\right)=\rho_{1}$.

In what follows we will consider sequences $\left(\rho_{1}, \ldots, \rho_{n}\right)$ for which the associated partition $\pi \in \mathcal{P}(n)$ has no crossings, i.e. $\pi \in \mathcal{N C}(n)$. We will be interested in the case when the label function is strictly increasing with respect to the two orderings: $\prec_{\pi}$ on blocks of $\pi$, and $\prec$ on elements of $\mathcal{X}$.

Definition 3.8 (bm-order on a non-crossing partition). Let $(\mathcal{X}, \preceq)$ be a partially ordered set and let $\left(\rho_{1}, \ldots, \rho_{n}\right)$ be a given sequence of elements from $\mathcal{X}$. Assume that the associated partition $\pi \sim\left(\rho_{1}, \ldots, \rho_{n}\right)$ has no crossings, i.e. $\pi=$ $\left(B_{1}, \ldots, B_{k}\right) \in \mathcal{N C}(n)$. We say that the sequence $\rho:=\left(\rho_{1}, \ldots, \rho_{n}\right)$ establishes a strict bm-order on the partition $\pi$ (notation $\rho \triangleleft \pi$ ) if for all $1 \leq i \neq j \leq k$ the condition $B_{i} \prec_{\pi} B_{j}$ implies that $l\left(B_{i}\right) \prec l\left(B_{j}\right)$. Similarly (and equivalently), we will say that the label sequence $\mu:=\left(l\left(B_{1}\right), \ldots, l\left(B_{k}\right)\right) \approx \pi$ establishes a strict bm-order on $\pi$ (notation: $\mu \triangleleft_{L} \pi$ ).

Remark 3.9. Observe that if $\max B_{i}<\min B_{j}$, then all three possibilities $l\left(B_{i}\right) \prec$ $l\left(B_{j}\right)$ or $l\left(B_{i}\right) \succ l\left(B_{j}\right)$ or $l\left(B_{i}\right) \nsim l\left(B_{j}\right)$ are allowed.

Example 3.10 (bm-order on a non-crossing partition). To illustrate the definition, take $\rho_{j} \in \mathbf{I}=\mathbb{N}^{2} \subset \Pi=\mathbb{R}^{2}(0 \leq j \leq 3)$ with $\rho_{0}=(0,0), \rho_{1}=(0,1), \rho_{2}=(1,0)$, $\rho_{3}=(1,1)$. These four elements constitute the discrete interval $\left[\rho_{0}, \rho_{3}\right]_{\mathbf{I}}$. Then $\rho_{1} \nsim \rho_{2}$ are incomparable, $\rho_{0} \prec \rho_{1}, \rho_{2}, \rho_{3}$ and $\rho_{0}, \rho_{1}, \rho_{2} \prec \rho_{3}$. Let $\pi \in \mathcal{N C}(6)$ be defined by the ordered blocks $B_{1}=\{1,6\}, B_{2}=\{2,3\}$ and $B_{3}=\{4,5\}$ then $B_{1} \prec_{\pi} B_{2}$ and $B_{1} \prec_{\pi} B_{3}$. Consider what are the possible bm-orderings on $\pi$ by the elements $\left\{\rho_{0}, \rho_{1}, \rho_{2}, \rho_{3}\right\}$ i.e. which sequences $\left(\rho_{j_{1}}, \rho_{j_{2}}, \ldots, \rho_{j_{6}}\right)$ establish strict bm-order on $\pi$ (with $0 \leq j_{1}, \ldots, j_{6} \leq 3$ ). The trivial condition is that it must be $j_{1}=j_{6}, j_{2}=j_{3}$ and $j_{4}=j_{5}$ since $\pi \sim\left(\rho_{j_{1}}, \rho_{j_{2}}, \ldots, \rho_{j_{6}}\right)$; hence the sequence must be of the form $\left(\rho_{j_{1}}, \rho_{j_{2}}, \rho_{j_{2}}, \rho_{j_{3}}, \rho_{j_{3}}, \rho_{j_{1}}\right)$ with $\rho_{j_{1}} \prec \rho_{j_{2}}$ and $\rho_{j_{1}} \prec \rho_{j_{3}}$. Thus the 
label $l\left(B_{1}\right)=\rho_{j_{1}}$ must have at least two elements bigger w.r.t. the partial order, so it must be $\rho_{j_{1}}=l\left(B_{1}\right)=\rho_{0}$. In the following table we present all 6 possible labellings.

\begin{tabular}{|l|l|l|l|l|l|l|}
\hline$l\left(B_{1}\right)$ & $\rho_{0}$ & $\rho_{0}$ & $\rho_{0}$ & $\rho_{0}$ & $\rho_{0}$ & $\rho_{0}$ \\
\hline$l\left(B_{2}\right)$ & $\rho_{1}$ & $\rho_{2}$ & $\rho_{1}$ & $\rho_{3}$ & $\rho_{2}$ & $\rho_{3}$ \\
\hline$l\left(B_{3}\right)$ & $\rho_{2}$ & $\rho_{1}$ & $\rho_{3}$ & $\rho_{1}$ & $\rho_{3}$ & $\rho_{2}$ \\
\hline
\end{tabular}

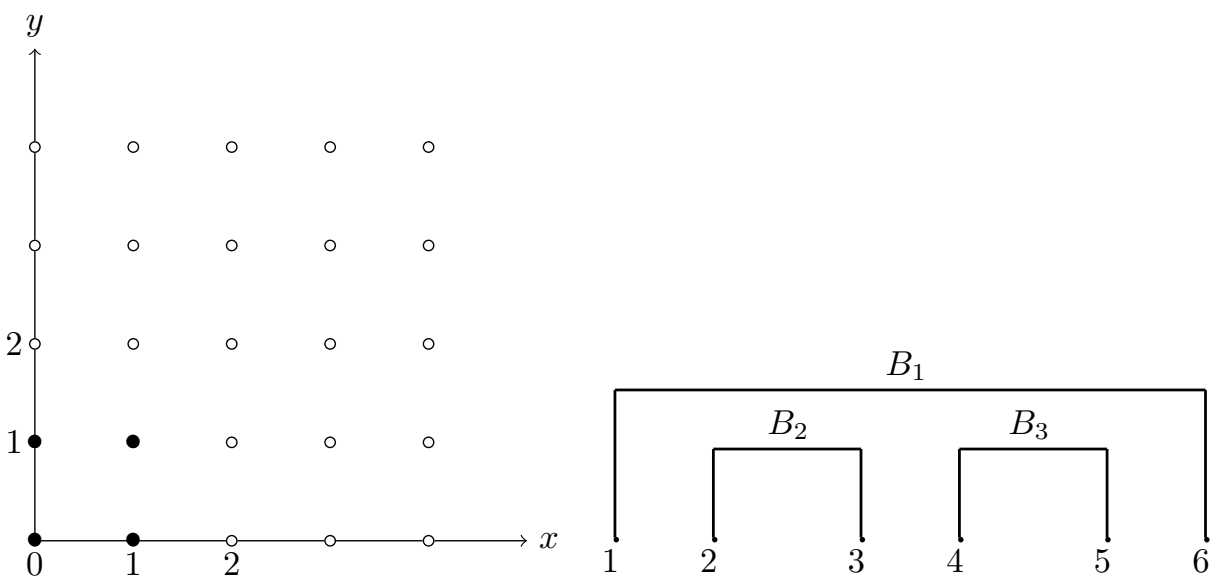

For $\pi \sim\left(\rho_{1}, \rho_{2}, \ldots, \rho_{n}\right)$, we construct a maximal non-crossing and strictly bmordered refinement $\sigma$ of $\pi$. For this purpose define the following relation on $[n]$ : $i \sim j$ provided $\rho_{i}=\rho_{j}$ and $\rho_{k} \succeq \rho_{i}$ for all $k$ between $i$ and $j(i, j, k \in[n])$. Then this is an equivalence relation on $[n]$. Denote by $\sigma$ the set of the equivalence classes, then $\sigma$ is a partition of [n]. Observe that if $B \in \sigma$ is a block, $i, j \in B$ and $i<k<j$, then either $\rho_{k}=\rho_{i}$, in which case $k \in B$, or $\rho_{i} \prec \rho_{k}$ and then $k \notin B$.

In the next lemma we describe properties of the partition $\sigma$. In particular we prove that it is the largest non-crossing strictly bm-ordered refinement of $\pi$.

Lemma 3.11. The partition $\sigma$ is a non-crossing, strictly bm-ordered refinement of $\pi$ and for every non-crossing strictly bm-ordered refinement $\nu$ of $\pi, \nu$ is a refinement of $\sigma$.

Proof: First we prove that $\sigma$ is non-crossing. Otherwise, there would be two equivalence classes (blocks) $B_{1} \neq B_{2}$ of $\sigma$, and elements $a<b<c<d$ with $a, c \in B_{1}$, $b, d \in B_{2}$ and $\rho_{a}=\rho_{c} \neq \rho_{b}=\rho_{d}$. Then, by definition of the equivalence relation $\sim$, it would have been $\rho_{a}=\rho_{c} \prec \rho_{b}=\rho_{d}$ and also $\rho_{c}=\rho_{d} \prec \rho_{b}=\rho_{a}$, which is a contradiction.

Observe that $\sigma$ is a refinement of $\pi$, since $\pi$ is defined by the relation: $i \sim_{\pi} j$ if $\rho_{i}=\rho_{j}$, and $\sigma$ is defined by adding one more condition to this relation.

To see that $\sigma$ is strictly bm-ordered, we use the definition of $\sigma$. Namely, if two blocks satisfy $B_{1} \prec_{\sigma} B_{2}$, with $B_{1}=\left\{i_{1}<\cdots<i_{r}\right\}, B_{2}=\left\{j_{1}<\cdots<j_{s}\right\}$, and with the labels $l\left(B_{1}\right)=\xi, l\left(B_{2}\right)=\eta$, then $\xi=\rho_{i_{p}} \prec \rho_{j_{q}}=\eta$ for all $1 \leq p \leq r$, $1 \leq q \leq s$. This means that $l\left(B_{1}\right) \prec l\left(B_{2}\right)$, so that $\sigma$ is strictly bm-ordered.

Now we prove that any strictly bm-ordered refinement $\nu$ of $\pi$ is a refinement of $\sigma$. For this purpose assume that a block $B \in \nu$ is contained in a block $B_{1} \in \pi$. By definition, the labels of these two blocks are equal: $l(B)=l\left(B_{1}\right)$. Since $\nu$ is non-crossing strictly bm-ordered, any block $B^{\prime} \in \nu$ which satisfies $B \prec{ }_{\nu} B^{\prime}$ must 
have greater label: $l(B) \prec l\left(B^{\prime}\right)$. This means that $B$ satisfies: if $i<j \in B$, then $\rho_{i}=\rho_{j}=l(B)$ and if $i<k<j$ then $\rho_{i} \prec \rho_{k}$. However, it follows from the definition of $\sigma$, that it consists of blocks which are maximal with this property - the blocks contain all such elements $i, j$ and $B$ contains only some of them. In other words, if $B=\left\{i_{1}<\cdots<i_{r}\right\}$ and $i_{p}<k<i_{p+1}$ for some $1 \leq p \leq r-1$, then $l(B)=\rho_{i_{1}}=\cdots=\rho_{i_{r}} \prec \rho_{k}$, but there is a block $B^{\prime \prime} \in \sigma$ which necessarily contains all elements $i_{1}, \ldots, i_{r}$, and possibly some other, defined by the equivalence relation $\sim$. Namely, $B^{\prime \prime}=\left\{j \in[n]: \rho_{j}=l(B)\right\} \supset B$ and for any $j_{1}<j_{2} \in B^{\prime \prime}$ and any $j_{1}<k<j_{2}$ it must be $\rho_{j_{1}}=\rho_{j_{2}} \prec \rho_{k}$. This shows that $\nu$ is a refinement of $\sigma$.

In the following theorem we describe the details of the algorithm which produces the refinement $\tau$ of $\pi$, described in Remark 2.3. We prove that $\tau$ is a coarsest (i.e. maximal) strictly bm-ordered refinement of $\pi$. The refinement $\tau$ turns out to be exactly the same partition as the partition $\sigma$ of Lemma 3.11. This will imply that the algorithm produces the unique maximal (i.e. largest) strictly bm-ordered non-crossing refinement of $\pi$.

Theorem 3.12. Let $\pi \sim\left(\rho_{1}, \rho_{2}, \ldots, \rho_{n}\right)$ be the partition of $[n]$ associated with a sequence of elements $\rho_{1}, \rho_{2}, \ldots, \rho_{n} \in \mathcal{X}$ (as in Definition 3.2) and let $\tau$ be the refinement of $\pi$ described in the Remark 2.3. Then $\tau$ is uniquely determined, and it is the coarsest possible refinement of $\pi$ such that $\tau$ is non-crossing and the labelling $l$ establishes a strict bm-order on $\tau$. In other words, $\tau$ is a non-crossing partition of $[n]$ which is strictly bm-ordered by $l$. Moreover, we have that $\tau=\sigma$ described in Lemma 3.11, so it is the unique coarsest non-crossing strictly bm-ordered refinement of $\pi$.

Proof: We recall the algorithm of Remark 2.3 that produces at least one possible refinement $\tau$ of $\pi$. The uniqueness of such $\tau$ follows from the proofs of Wysoczański (2010, Lemma 2.3 and Lemma 2.4).

We shall define restrictions of $\pi$ to subsets of $[n]$, from which the refinement $\tau$ will be constructed.

Denote $\pi_{0}:=\pi$ and $J_{0}:=[n]$ and suppose there is an interval block $B_{0}=$ $\left[a_{0}, b_{0}\right] \in \pi$ for $2 \leq a \leq b \leq n-1$, such that the triplet of labels $(\mu, \xi, \eta)$, defined by $\mu:=\rho_{a_{0}-1}, \xi:=l(B)=\rho_{a_{0}}=\cdots=\rho_{b_{0}}$ and $\eta:=\rho_{b_{0}+1}$, satisfy the [BM1] assumption: either $\mu \prec \xi \succ \eta$ or $\mu \nsim \xi \succ \eta$ or $\mu \prec \xi \nsim \eta$. Then, by definition, $B_{0}$ is a block of $\tau$. Now define $\pi_{1}:=\pi_{0} \backslash B_{0} \in \mathcal{P}\left(J_{1}\right)$, where $\left.J_{1}:=J_{0} \backslash B_{0}=[n] \backslash[a), b_{0}\right]$, then $\pi_{1}$ is a subpartition of $\pi_{0}$ to which the same procedure is applied. By induction we construct a finite sequence of partitions $\pi_{r}:=\pi_{r-1} \backslash B_{r-1} \in \mathcal{P}\left(J_{r}\right)$, for $r=$ $1,2, \ldots$, and $J_{r}:=J_{r-1} \backslash B_{r-1}, I_{r}:=\left[a_{r}, b_{r}\right]$, and blocks $B_{r} \in \pi_{r}$, which constitute blocks of $\tau$. This procedure stops after, say, $m$ steps, producing blocks $B_{1}, \ldots, B_{m}$ of $\tau$, when there is no more interval block $B_{m+1}$ in $\pi_{m+1}:=\pi_{m} \backslash B_{m} \in \mathcal{P}\left(J_{m+1}\right)$, for which there is a triplet of labels, which satisfies the [BM1] assumption.

In a similar manner as in the proof of Wysoczański (2010, Lemma 2.3) one can show that the partition $\pi_{m+1}$ consists of interval blocks only, so $\pi_{m+1}:=$ $\left\{B_{m+1}, \ldots, B_{k}\right\}$ with $\max B_{i}<\min B_{i+1}$ for $m+1 \leq i \leq k-1$, and their labels satisfy

$$
l\left(B_{m+1}\right) \succ \cdots \succ l\left(B_{u}\right) \nsim \cdots \nsim l\left(B_{v}\right) \prec \cdots \prec l\left(B_{k}\right),
$$

as in the assumption of [BM2], for some $m+1 \leq u \leq v \leq k$ (allowing also $u=m+1$ or $u=v$ or $v=k$ ). Moreover, the arguments in Wysoczański (2010, Lemma 2.3 and 
Lemma 2.4) show that any order of choosing blocks $B_{0}, B_{1}, \ldots, B_{m}, B_{m+1}, \ldots, B_{k}$, gives the same result, i.e. these blocks of the partition $\tau$ are uniquely determined.

Of course, if there is no such interval block $B_{0}$ in $\pi_{0}=\pi$, for which a [BM1]triplet exists, then $\pi=\pi_{m+1}$ is the interval partition as above.

We conclude this part of the proof with the statement that the above algorithm uniquely determines the partition

$$
\tau:=\left\{B_{0}, B_{1}, \ldots, B_{m}, B_{m+1}, \ldots, B_{k}\right\} .
$$

Now we show that $\tau$ has no crossings and the blocks of $\tau$ are strictly bm-ordered. For this purpose consider the step which produced the block $B_{m}$. Since $B_{m}$ is an interval block, it had to be either inside ${ }^{2}$ a block $B_{i}$ of $\pi_{m}$ or between ${ }^{3}$ two blocks $B_{i}, B_{i+1}$ of $\pi_{m}$. In the first case, we must have $l\left(B_{i}\right) \prec l\left(B_{m}\right)$, otherwise the algorithm would not be applicable ([BM1] could not be applied). Of course, it could not be $l\left(B_{i}\right)=l\left(B_{m}\right)$, since the algorithm applies to a maximal interval block $B_{m}$. Thus we have a strict bm-order between the two blocks. In the second case $\left(B_{m}\right.$ between two blocks $B_{i}$ and $B_{i+1}$ of $\left.\tau^{\prime}\right)$, it must have been either $l\left(B_{i}\right) \prec l\left(B_{m}\right) \succ l\left(B_{i+1}\right)$ or $l\left(B_{i}\right) \nsim l\left(B_{m}\right) \succ l\left(B_{i+1}\right)$ or $l\left(B_{i}\right) \prec l\left(B_{m}\right) \nsim l\left(B_{i+1}\right)$. This, however, does not provide any condition related to bm-order of blocks.

In general, at each backward step of the algorithm we get strict bm-order if the removed interval block was inside another block. By induction, this proves that the partition $\tau$ has no crossings (since blocks are intervals) and is strictly bm-ordered. Therefore, by Lemma 3.11, we have that $\tau$ is a refinement of $\sigma$.

We will prove that, in fact, $\tau=\sigma$. For this purpose it suffices to show that $\tau$ cannot be a strict refinement $\sigma$. Assume to the contrary that $\tau$ is a strict refinement of $\sigma$. Then there is a block $B^{\prime} \in \sigma$ and two interval blocks $B_{i}, B_{j} \in \tau$ which are subblocks $B_{i}, B_{j} \subset B^{\prime}$, in particular $l\left(B_{i}\right)=l\left(B_{j}\right)=l\left(B^{\prime}\right)$. Without loss of generality, we can assume that $b_{i}:=\max B_{i}<a_{j}:=\min B_{j}$ and that there is no other block $B_{l} \in \tau$ between ${ }^{4} B_{i}$ and $B_{j}$, which is a sub-block of $B^{\prime}$ (although there might be other blocks of $\tau$, which are contained in $B^{\prime}$ ). Consider the subpartition $\tau_{0} \subset \tau$ obtained by the restriction of $\tau$ to the interval $\left[b_{i}+1, a_{j}-1\right]$. This interval must be nonempty (i.e. $b_{i}+1 \leq a_{j}-1$ ), otherwise $B_{i} \cup B_{j}$ would be one interval block of $\tau$. Since $\tau$ is strictly bm-ordered, its subpartition $\tau_{0}$ is so as well.

Observe that since $\sigma$ has no crossings, it cannot contain a block with elements both inside and outside the interval $\left[b_{i}+1, a_{j}-1\right]$. As $\tau$ is a refinement of $\sigma$, it inherits this property.

Each block $B^{\prime \prime} \in \tau_{0} \subset \tau$ is inside the block $B^{\prime} \in \sigma$, hence $l\left(B^{\prime \prime}\right) \succ l\left(B^{\prime}\right)$ and thus $l\left(B_{i}\right) \prec l\left(B^{\prime \prime}\right) \succ l\left(B_{j}\right)$. Therefore the algorithm cannot be applied neither to $B_{i}$ nor to $B_{j}$ until it is applied to all the blocks of $\tau_{0}$. However, if the algorithm allowed to remove $\tau_{0}$, then it would mean that the blocks $B_{i}$ and $B_{j}$ would be adjacent and they both would form one interval block of $\tau$ subject to the algorithm. This contradicts the assumption that these were two different blocks of $\tau$ and shows that $\tau$ cannot be a refinement of any non-crossing strictly bm-ordered partition. Consequently, $\tau$, given by the algorithm, is the coarsest non-crossing strictly bmordered refinement of $\pi$. In particular, $\tau=\sigma$, and it is the largest element in this

\footnotetext{
$2^{2}$ by this we mean that if $B_{m}=\left[a_{m}, b_{m}\right]$ then $B_{i}=\left[a_{i}, a_{m}-1\right] \cup\left[a_{m}+1, b_{i}\right]$.

$3_{\text {if }} B_{m}=\left[a_{m}, b_{m}\right]$ then $B_{i}=\left[a_{i}, a_{m}-1\right]$ and $B_{i+1}=\left[b_{m}+1, b_{i}\right]$

$4_{\text {such that }} a_{i}<a_{l} \leq b_{l}<a_{j}$
} 
particular class of non-crossing strictly bm-ordered partitions, which are refinements of a given $\pi \sim\left(\rho_{1}, \rho_{2}, \ldots, \rho_{n}\right)$. This finishes the proof of the theorem.

The following corollary will be the main tool in proving our main results.

Corollary 3.13. Let $\left\{\mathcal{A}_{\rho}: \rho \in \mathcal{X}\right\}$ be a family of bm-independent subalgebras in $(\mathcal{A}, \varphi)$ and let $a_{j} \in \mathcal{A}_{\rho_{j}}$ for $1 \leq j \leq n$. Let $\pi \sim\left(\rho_{1}, \rho_{2}, \ldots, \rho_{n}\right)$ be the associated partition of $[n]$ and let $\tau$ be the coarsest non-crossing refinement of $\pi$ (as in Theorem 3.12) with blocks $\tau=\left\{B_{1}, \ldots, B_{k}\right\}$. Then

$$
\varphi\left(a_{1} a_{2} \ldots a_{n}\right)=\prod_{i=1}^{k} \varphi\left(\prod_{j \in B_{i}} a_{j}\right),
$$

where $\varphi\left(\prod_{j \in B_{i}} a_{j}\right):=\varphi\left(a_{j_{1}} \cdots a_{j_{r}}\right)$ if $B_{i}=\left(j_{1}, \ldots, j_{r}\right)$ with $j_{1}<\cdots<j_{r}$.

Proof: The algorithm for passing from $\pi$ to $\tau$ starts with choosing an interval block $B=(p, \ldots, p+s) \in \pi$ with $\rho_{p}=\cdots=\rho_{p+s}=\rho$ with $\xi:=\rho_{p-1} \neq \rho \neq \rho_{p+s+1}=: \eta$ as a first block of $\tau$ and then removing it and considering $\tau \backslash B$. This is done if the indices $\xi, \rho, \eta$ satisfy the assumption of [BM1]. This corresponds to taking the product $a:=a_{p} \cdots a_{p+s}$ and getting out the term $\varphi\left(a_{\rho}\right):=\varphi\left(a_{p} \cdots a_{p+s}\right)=$ $\varphi\left(\prod_{j \in B} a_{j}\right)$. As shown in the proof of Theorem 3.12, this leads to the subpartition $\tau^{\prime}=\left(B_{m+1}, \ldots, B_{k}\right)$ of $\tau$, which satisfies $\max B_{i}<\min B_{i+1}$ for $m+1 \leq i \leq k-1$ and the assumption of [BM2]: $l\left(B_{m+1}\right) \succ \cdots \succ l\left(B_{u}\right) \nsim \cdots \nsim l\left(B_{v}\right) \prec \cdots \prec l\left(B_{k}\right)$. This corresponds to taking the product $\varphi\left(\prod_{j \in B_{m+1}} a_{j}\right) \cdots \varphi\left(\prod_{j \in B_{k}} a_{j}\right)$, where $\varphi\left(\prod_{j \in B_{i}} a_{j}\right)=\varphi\left(a_{j_{1}} \cdots a_{j_{r}}\right)$ if $B_{i}=\left(j_{1}, \ldots, j_{r}\right)$ with $j_{1}<\cdots<j_{r}$. This proves the Corollary.

Notation 3.14. By $\pi \stackrel{b m}{\longrightarrow} \tau$ we denote that $\tau$ is the coarsest strictly bm-ordered non-crossing refinement of $\pi \sim\left(\rho_{1}, \ldots, \rho_{n}\right)$.

\section{Main results}

In this section, we give the detailed formulation of our bm-LSN. In particular, we describe the function $V(\pi)$ that appeared in Theorem 2.4. We prove the bm-LSN using certain combinatorial objects, of which the most important is the set of all bm-orders on a non-crossing partition with labels from a (finite, discrete) interval $[0, \xi]_{\mathbf{I}} \subset \mathbf{I}$.

4.1. Formulation of bm-LSN. We first introduce the set of all strict bm-orders on a given non-crossing partition, with labels taken from a given interval.

Definition 4.1. For $\pi=\left(B_{1}, \ldots, B_{k}\right) \in \mathcal{N C}(n ; k)$ and $\xi \in \Pi$ we define

$$
\operatorname{BMO}(\pi, \xi):=\left\{\mu=\left(\mu_{1}, \ldots, \mu_{k}\right): \mu_{j}=l\left(B_{j}\right) \in[0, \xi]_{\mathbf{I}} 1 \leq j \leq k \text {, and } \mu \triangleleft_{L} \pi\right\} .
$$

We will denote the cardinality of this set by $|\operatorname{BMO}(\pi, \xi)|$.

Definition 4.1 does not require that $\xi \in \mathbf{I}$, only that the labels are taken from the finite set $[0, \xi]_{\mathbf{I}}$.

Our main technical goal will be to describe the asymptotic behaviour of these cardinalities for the three classes of positive symmetric cones, as $\xi \stackrel{\Pi}{\rightarrow} \infty$. For 
this purpose we consider the following decomposition. If $\pi \in \mathcal{N C}(n)$ has $m \leq$ $n$ outer blocks (denoted $\left\{B_{1}^{o}, \ldots, B_{m}^{o}\right\}$ ) with $a_{j}=\min B_{j}^{o}$ and $b_{j}=\max B_{j}^{o}$ for $1 \leq j \leq m$, then the restriction $\pi_{j}:=\pi \uparrow_{\left[a_{j}, b_{j}\right]_{\mathbb{N}}}$ of $\pi$ to the discrete interval $\left[a_{j}, b_{j}\right]_{\mathbb{N}}:=\left[a_{j}, b_{j}\right] \cap \mathbb{N} \subset[n]$ will be considered as a subpartition of $\pi$ with exactly one outer block $B_{j}^{o}$. Then we will use the notation $\pi_{j}^{\prime}:=\pi_{j} \backslash B_{j}^{o}$, so that the number of blocks in $\pi$ satisfies

$$
\begin{aligned}
b(\pi) & =\sum_{j=1}^{m} b\left(\pi_{j}\right), \\
b\left(\pi_{j}\right) & =b\left(\pi_{j}^{\prime}\right)+1 .
\end{aligned}
$$

Notation 4.2. Let $\pi=\left(B_{1}, \ldots, B_{k}\right)$ be the ordered blocks of a partition $\pi \in$ $\mathcal{N C}(n, k)$ (in particular, $1 \in B_{1}$ and the block $B_{1}$ is outer). The partition $\pi^{\prime}:=$ $\pi \backslash\left\{B_{1}\right\}=\left(B_{2}, \ldots, B_{k}\right)$ satisfies $\pi^{\prime} \in \mathcal{N C}\left([n] \backslash B_{1}, k^{\prime}\right)$ where $k^{\prime}:=k-1<k$.

Notation 4.3. For $\pi=\left(B_{1}, \ldots, B_{k}\right) \in \mathcal{N C}(n ; k)$ let $m:=\max \left(B_{1}\right)+1 \geq 2$ (i.e. $m-1=\max B_{1}$ is the right endpoint of the block $\left.B_{1}\right)$. Then $B_{1} \subset[m-1]$ and we define the following two restrictions of $\pi^{\prime}$ :

(1) $\pi_{1}^{\prime}:=\pi^{\prime} \uparrow_{[1, m-1]} \in \mathcal{N C}\left([m-1] \backslash B_{1}, k_{1}^{\prime}\right)$ is the restriction of $\pi^{\prime}$ to the interval $[m-1]=\{1, \ldots, m-1\}$, and $k_{1}^{\prime}:=b\left(\pi_{1}^{\prime}\right)$;

(2) $\pi_{2}^{\prime}:=\pi^{\prime} \uparrow_{[m, n]}=\pi \uparrow_{[m, n]} \in \mathcal{N C}\left([m, n], k_{2}^{\prime}\right)$ is the restriction of $\pi^{\prime}$ to the interval $[m, n]=\{m, \ldots, n\}$ and $k_{2}^{\prime}:=b\left(\pi_{2}^{\prime}\right)$.

In particular, if $n_{1}^{\prime}:=m-1-\left|B_{1}\right|$ and $n_{2}^{\prime}:=n-m+1$, then $k_{1}^{\prime}+k_{2}^{\prime}+1=k=b(\pi)$ and $n_{1}^{\prime}+n_{2}^{\prime}=n-\left|B_{1}\right|$. In the following picture we present the decomposition $\pi=B_{1} \cup \pi_{1}^{\prime} \cup \pi_{2}^{\prime}$ with $\pi_{1}^{\prime}:=\pi_{1,1}^{\prime} \cup \pi_{1,2}^{\prime} \cup \cdots \cup \pi_{1, s}^{\prime}$ :

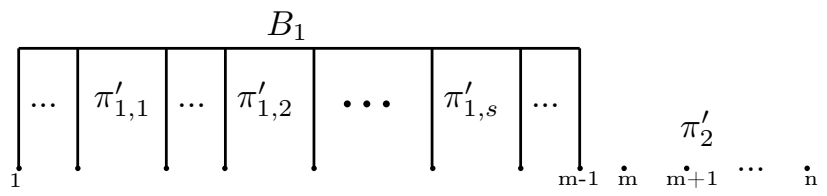

where the vertical lines denote the (possible ends of) intervals included in $B_{1}$. The notation we use for the decomposition $\pi_{1}^{\prime}=\pi_{1,1}^{\prime} \cup \pi_{1,2}^{\prime} \cup \cdots \cup \pi_{1, s}^{\prime}$ has the following meaning. Let $B_{1}=\left\{1=t_{0}<t_{1}<t_{2}<\cdots<t_{l}=m-1=\max B_{1}\right\}$ and let $1 \leq j_{1} \leq l$ be the minimal element for which $t_{j_{1}}-t_{j_{1}-1} \geq 2$, then $\pi_{1,1}^{\prime}:=\pi_{1}^{\prime} \uparrow_{\left[t_{j_{1}-1}+1, t_{j_{1}}-1\right]}$. Then, by induction, one defines in a similar way, indices $j_{2}<j_{3}<\cdots<j_{s}$ for which $t_{j_{r}}-t_{j_{r}-1} \geq 2(2 \leq r \leq s)$, and then $\pi_{1, r}^{\prime}:=\pi_{1}^{\prime} \Upsilon_{\left[t_{j_{r}-1}+1, t_{j_{r}}-1\right]}$.

Now we present the main result of the paper, the bm-LSN associated with the symmetric cones considered in this work.

Theorem 4.4 (bm-Laws of Small Numbers). Let $\Pi=\mathbb{R}_{+}^{d}, \Pi=\Lambda_{d}^{1}$ or $\Pi=$ $\operatorname{Symm}_{d}^{+}(\mathbb{R})$ and let $\mathbf{I} \subset \Pi$ be the respective discrete subset as in Definition 1.4. Let $(\mathcal{A}, \varphi)$ be a noncommutative probability space and let $\left\{X_{\rho}^{\xi} \in \mathcal{A}: \rho, \xi \in \mathbf{I}, 0 \preceq \rho \preceq \xi\right\}$ be an array of self-adjoint (noncommutative) random variables. Assume that

(1) for each $\xi \in \mathbf{I}$, the random variables $\left\{X_{\rho}^{\xi} \in \mathcal{A}: 0 \preceq \rho \preceq \xi, \rho \in \mathbf{I}\right\}$ are bm-independent (with respect to $\varphi$ ) 
(2) there exists a constant $\lambda>0$ such that for all $\rho \in \mathbf{I}$ and for all $n \in \mathbb{N}$

$$
\lim _{\xi \rightarrow \infty} \sup _{\rho \in[0, \xi]_{\mathbf{I}}}\left|v(\xi) \cdot \varphi\left(\left(X_{\rho}^{\xi}\right)^{n}\right)-\lambda\right|=0 .
$$

For $\xi \in \mathbf{I}$, define the partial sum $S_{\xi}:=\sum_{\rho \in[0, \xi] \mathbf{I}} X_{\rho}^{\xi}$. Then, for every $n \in \mathbb{N}$ the limit

$$
\lim _{\xi \rightarrow \infty} \varphi\left(\left(S_{\xi}\right)^{n}\right)=\sum_{\pi \in \mathcal{N} \mathcal{C}(n)} \lambda^{b(\pi)} V(\pi)
$$

exists, where the function $V:=V_{\Pi}$, defined on non-crossing partitions, depends on the positive cone and is given by the limit

$$
V(\pi)=\lim _{\xi \stackrel{\Pi}{\rightarrow} \infty} \frac{|\operatorname{BMO}(\pi, \xi)|}{v(\xi)^{b(\pi)}} .
$$

Here, $b(\pi)$ is the number of blocks in the partition $\pi$ and the limit exists for every partition $\pi \in \mathcal{N C}(n)$. Moreover, with the Notation 4.3, the function $V(\pi)$ is satisfies the following recursive formula:

(1) If $b(\pi)=1$, then $V(\pi)=1$.

(2) If $b(\pi) \geq 2$, then

$$
V(\pi)=\gamma_{k_{1}^{\prime}+1} V\left(\pi_{1}^{\prime}\right) V\left(\pi_{2}^{\prime}\right),
$$

where the sequence $\gamma_{n}$ is the volume characteristic sequence of the positive cone $\Pi$.

The details of the proofs of Theorem 4.4 for each class of positive cones will be presented in the next sections.

By induction, one can get the following corollary of Theorem 4.4.

Corollary 4.5. The function $V(\pi)$ satisfies the following recursive formula:

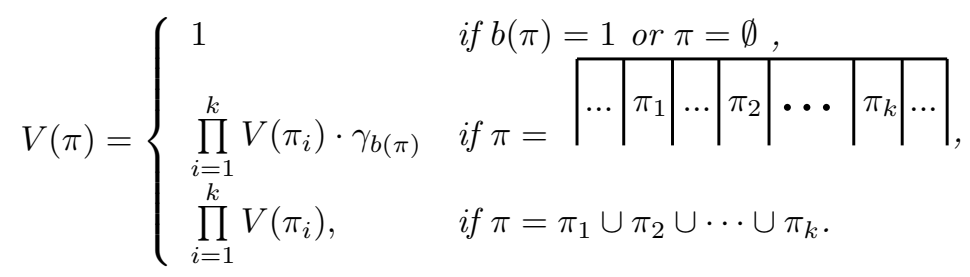

In the second case, the form of the partition $\pi$ is understood as in the Notation 4.3 (i.e. as if $\pi=\pi_{1}^{\prime} \cup B_{1}$ ). By writing $\pi=\pi_{1} \cup \pi_{2} \cup \cdots \cup \pi_{k}$ we mean a disjoint union of subpartitions $\pi_{s}:=\pi \uparrow_{\left[a_{s}, b_{s}\right]}$, for $1 \leq s \leq k$, where $\left[a_{s}, b_{s}\right] \subset[n], a_{s}+1=b_{s}$, $a_{1}=1, b_{s}=n$, and each $\pi_{s}$ is a partition with exactly one outer block (which could be a singleton as well if $a_{s}=b_{s}$ ).

4.2. Examples of moments for various $L S N$. For illustration, we compare the first four moments of the classical, free, monotone and some bm-LSN distributions (here we use the formula (1.2)). First we recall the classical, free and monotonic cases (observe that always the first two moments are $\lambda$ and $\lambda^{2}+\lambda$ ). 


$$
\begin{array}{ccc}
\text { Classical } & \text { Free } & \text { Monotone } \\
\lambda & \lambda & \lambda \\
\lambda^{2}+\lambda & \lambda^{2}+\lambda & \lambda^{2}+\lambda \\
\lambda^{3}+3 \lambda^{2}+\lambda & \lambda^{3}+3 \lambda^{2}+\lambda & \lambda^{3}+\frac{5}{2} \lambda^{2}+\lambda \\
\lambda^{4}+6 \lambda^{3}+7 \lambda^{2}+\lambda & \lambda^{4}+6 \lambda^{3}+6 \lambda^{2}+\lambda & \lambda^{4}+\frac{13}{3} \lambda^{3}+\frac{9}{2} \lambda^{2}+\lambda
\end{array}
$$

Now we present some of the bm-examples, using the volume characteristic formulas from the Example 1.3.

$$
\begin{array}{cc}
\text { bm-cases } \Pi=\mathbb{R}_{+}^{2} \text { and } \Pi=\Lambda_{1}^{1} & \text { bm-cases } \Pi=\Lambda_{2}^{1} \text { and } \Pi=\operatorname{Symm}_{2}^{+}(\mathbb{R}) \\
\lambda & \lambda \\
\lambda^{2}+\lambda & \lambda^{2}+\lambda \\
\lambda^{3}+\frac{9}{4} \lambda^{2}+\lambda & \lambda^{3}+\frac{222}{105} \lambda^{2}+\lambda \\
\lambda^{4}+\frac{65}{18} \lambda^{3}+\frac{15}{4} \lambda^{2}+\lambda & \lambda^{4}+\frac{10275}{3150} \lambda^{3}+\frac{702}{210} \lambda^{2}+\lambda
\end{array}
$$

\section{Combinatorial reduction}

The proof of Theorem 4.4 starts with several combinatorial reductions. First we will show that the limit (4.2) can be associated with a sum over non-crossing partitions $\pi \in \mathcal{N C}(n)$. Then we will argue that the only contributions to the sum that are nontrivial in the limit are described by the bm-labellings $\mu \triangleleft_{L} \pi$ for such partitions $\pi$. Next we will show that the limit can be equivalently computed as the limit (for $\xi \stackrel{\Pi}{\rightarrow} \infty$ ) of the ratio of the cardinality $|\operatorname{BMO}(\pi, \xi)|$ and the volume $v(\xi)^{k}$ if $\pi \in \mathcal{N C}(n, k)$. Finally, we will prove that the limit of this ratio exists and describes the combinatorial function $V(\pi)$ associated with the positive cone $\Pi$.

We start the proof with the observation that $\varphi\left(S_{\xi}^{n}\right)$ can be written as

$$
\begin{aligned}
\varphi\left(\left(S_{\xi}\right)^{n}\right) & =\sum_{\left.\left(\rho_{1}, \ldots, \rho_{n}\right) \in([0, \xi]]_{\mathbf{I}}\right)^{n}} \varphi\left(X_{\rho_{1}}^{\xi} X_{\rho_{2}}^{\xi} \ldots X_{\rho_{n}}^{\xi}\right) \\
& =\sum_{\pi \in \mathcal{P}(n)} \sum_{\pi \sim\left(\rho_{1}, \ldots, \rho_{n}\right) \in\left([0, \xi]_{\mathbf{I}}\right)^{n}} \varphi\left(X_{\rho_{1}}^{\xi} X_{\rho_{2}}^{\xi} \ldots X_{\rho_{n}}^{\xi}\right) \\
& =\sum_{k=1}^{n} \sum_{\pi \in \mathcal{P}(n ; k)} \sum_{\pi \sim\left(\rho_{1}, \ldots, \rho_{n}\right) \in\left([0, \xi]_{\mathbf{I}}\right)^{n}} \varphi\left(X_{\rho_{1}}^{\xi} X_{\rho_{2}}^{\xi} \ldots X_{\rho_{n}}^{\xi}\right) .
\end{aligned}
$$

Given $1 \leq k \leq n$, consider a partition with ordered blocks $\pi=\left(B_{1}, \ldots, B_{k}\right) \in$ $\mathcal{P}(n ; k), b(\pi)=k$, then the block cardinalities satisfy $1 \leq\left|B_{j}\right|=n_{j} \leq n$ and $n_{1}+$ $\cdots+n_{k}=n$. Assume that $\pi$ is associated with a sequence $\left(\rho_{1}, \ldots, \rho_{n}\right) \in\left([0, \xi]_{\mathbf{I}}\right)^{n}$ and denote the block labels by $l\left(B_{j}\right)=\mu_{j}$, so that $\left(l\left(B_{1}\right), \ldots, l\left(B_{k}\right)\right)=\left(\mu_{1}, \ldots, \mu_{k}\right)$. Then, by the conditions for bm-independence, in particular by Corollary 3.13, the term $\varphi\left(X_{\rho_{1}}^{\xi} X_{\rho_{2}}^{\xi} \ldots X_{\rho_{n}}^{\xi}\right)$ is equal to the product of marginals

$$
\varphi\left(X_{\rho_{1}}^{\xi} X_{\rho_{2}}^{\xi} \ldots X_{\rho_{n}}^{\xi}\right)=C\left(\mu_{1}\right) \cdots C\left(\mu_{k}\right)=: C(\mu), \quad \text { for } \quad \mu:=\left(\mu_{1}, \ldots, \mu_{k}\right),
$$

where each term $C\left(\mu_{j}\right), 1 \leq j \leq k$, is the product of (some) moments of the random variables $X_{\mu_{j}}^{\xi}$, and it is worthwhile to observe the connection with the 
coarsest non-crossing strictly bm-ordered refinement $\tau$ of $\pi$ :

$$
C\left(\mu_{j}\right):=\varphi\left(\left(X_{\mu_{j}}^{\xi}\right)^{s_{1}}\right) \cdots \varphi\left(\left(X_{\mu_{j}}^{\xi}\right)^{s_{r(j)}}\right)=\prod_{\substack{B \in \tau \\ l(B)=\mu_{j}}} \varphi\left(\left(X_{\mu_{j}}^{\xi}\right)^{|B|}\right),
$$

where

$$
r\left(\mu_{j}\right)=\left|\left\{B \in \tau: l(B)=\mu_{j}\right\}\right|=\left|\tau \cap B_{j}\right|
$$

denotes the number of blocks of $\tau$ which comprise the block $B_{j}$ of $\pi$, and finally

$$
r(\mu):=r\left(\mu_{1}\right)+\cdots+r\left(\mu_{k}\right)=|\tau| .
$$

factors $\varphi\left(\left(X_{\mu_{j}}^{\xi}\right)^{s_{i}}\right)\left(1 \leq i \leq r\left(\mu_{j}\right)\right)$ on the right-hand side of (5.2), for the label $\mu_{j}=l\left(B_{j}\right)$. Moreover, given a label $\mu_{j}$, the exponents $1 \leq s_{1}, \ldots, s_{r\left(\mu_{j}\right)}$ depend on $j$ and satisfy $s_{1}+\cdots+s_{r(j)}=n_{j}=\left|B_{j}\right|$. This notation will be used further on.

Lemma 5.1. For each positive symmetric cone $\Pi$ under consideration we have

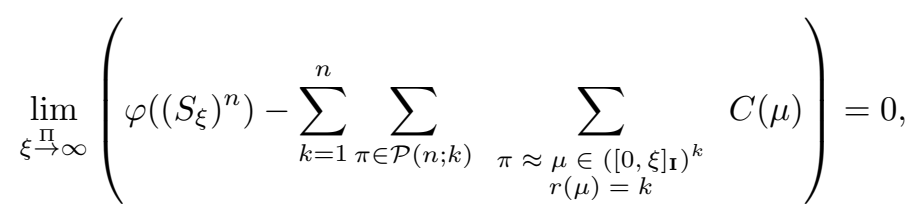

where

$$
C(\mu)=\prod_{j=1}^{k} \varphi\left(\left(X_{\mu_{j}}^{\xi}\right)^{\left|B_{j}\right|}\right)
$$

The third summation in (5.4) is over all label sequences $\mu:=\left(\mu_{1}, \ldots, \mu_{k}\right) \in\left([0, \xi]_{\mathbf{I}}\right)^{k}$ associated with a given partition $\pi$ such that $r(1)=\cdots=r(k)=1$ and $C\left(\mu_{j}\right)=$ $\varphi\left(\left(X_{\mu_{j}}^{\xi}\right)^{n_{j}}\right), n_{j}:=\left|B_{j}\right|(c f .(5.2))$.

Proof: By the assumption (2) of Theorem 4.4 we know that for each $1 \leq j \leq k$

$$
\lim _{\xi \rightarrow \infty} v(\xi)^{r(j)} C\left(\mu_{j}\right)=\lim _{\xi \rightarrow \infty}\left[v(\xi) \varphi\left(\left(X_{\mu_{j}}^{\xi}\right)^{s_{1}}\right)\right] \ldots\left[v(\xi) \varphi\left(\left(X_{\mu_{j}}^{\xi}\right)^{s_{r(j)}}\right)\right]=\lambda^{r(j)},
$$

hence we get

$$
\lim _{\xi \rightarrow \infty} v(\xi)^{r} \varphi\left(X_{\rho_{1}}^{\xi} X_{\rho_{2}}^{\xi} \ldots X_{\rho_{n}}^{\xi}\right)=\lim _{\xi \rightarrow \infty} v(\xi)^{r(\mu)} C(\mu)=\prod_{j=1}^{k} \lim _{\xi \rightarrow \infty} v(\xi)^{r(j)} C\left(\mu_{j}\right)=\lambda^{r(\mu)} .
$$

Observe that for any $\mu:=\left(\mu_{1}, \ldots, \mu_{k}\right)$ we have $r(\mu):=r(1)+\cdots+r(k) \geq k$ and that $r(\mu)=k$ if and only if $r(1)=\cdots=r(k)=1$. Thus, if $r(\mu) \geq k+1$, we have that

$$
\lim _{\xi \rightarrow \infty} v(\xi)^{k} C(\mu)=\lim _{\xi \rightarrow \infty} v(\xi)^{r(\mu)} C(\mu) \cdot \frac{v(\xi)^{k}}{v(\xi)^{r(\mu)}}=\lambda \cdot 0=0 .
$$

Therefore, given $\pi \in \mathcal{P}(n ; k)$, we split the summation

$$
\begin{aligned}
& \sum_{\pi \sim\left(\rho_{1}, \ldots, \rho_{n}\right) \in\left([0, \xi]_{\mathbf{I}}\right)^{n}} \varphi\left(X_{\rho_{1}}^{\xi} X_{\rho_{2}}^{\xi} \ldots X_{\rho_{n}}^{\xi}\right)=\sum_{\pi \approx\left(\mu_{1}, \ldots, \mu_{k}\right) \in\left([0, \xi]_{\mathbf{I}}\right)^{k}} C\left(\mu_{1}\right) \ldots C\left(\mu_{k}\right)
\end{aligned}
$$

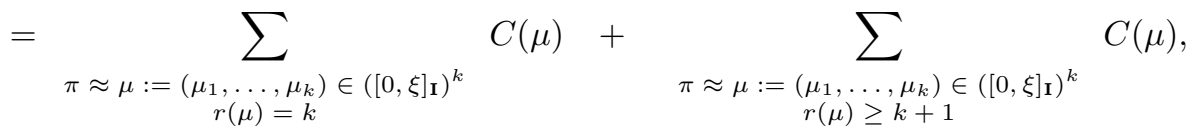


and show by a quantitative argument that the second sum (with $r(\mu) \geq k+1$ ) tends to zero. For this purpose, we use (5.6) to observe that for every $\mu \approx \pi$ with $r(\mu) \geq k+1$ we have the uniform estimate (for sufficiently large $\xi \stackrel{\Pi}{\rightarrow} \infty$ )

$$
C(\mu) \leq 2\left(\frac{\lambda}{v(\xi)}\right)^{r(\mu)} \leq 2\left(\frac{\lambda}{v(\xi)}\right)^{k+1}
$$

Therefore,

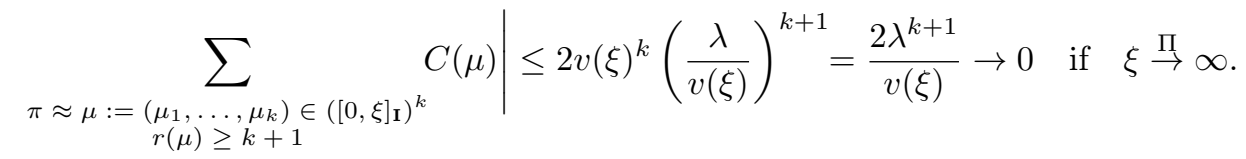

Hence we obtain the following approximate equality (for $\xi \stackrel{\Pi}{\rightarrow} \infty$ ):

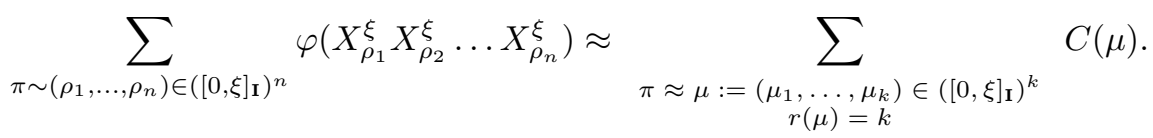

This means that, for given $1 \leq k \leq n$, the only nontrivial contribution to the limit $\lim _{\bar{\Pi}} \varphi\left(\left(S_{\xi}\right)^{n}\right)$ in Theorem 4.4 is from the terms $C(\mu)$, for which $\mathcal{P}(n ; k) \ni \pi \approx \mu=$ $\xi \stackrel{\Pi}{\rightarrow}$

$\left(\mu_{1}, \ldots, \mu_{k}\right)$ with $r(1)=\cdots=r(k)=1$, i.e. it must be $C\left(\mu_{j}\right)=\varphi\left(\left(X_{\mu_{j}}^{\xi}\right)^{\left|B_{j}\right|}\right)$ if $l\left(B_{j}\right)=\mu_{j}(j=1, \ldots, k)$ and hence

$$
C(\mu)=\prod_{j=1}^{k} \varphi\left(\left(X_{\mu_{j}}^{\xi}\right)^{\left|B_{j}\right|}\right) .
$$

Lemma 5.1 shows than the only terms that contribute to the limit as $\xi \stackrel{\Pi}{\rightarrow} \infty$ satisfy $r(1)=\cdots=r(k)=1$ and this means that $\pi=\tau$ where $\pi \stackrel{b m}{\longrightarrow} \tau$. By Theorem 3.12 and Corollary 3.13, this happens if and only if $\pi$ is non-crossing and the labels produce a strict bm-ordering on it. Hence, in order to compute the limit (5.4), we can restrict to summation over those sequences $\rho_{1}, \ldots, \rho_{n} \in[0, \xi]_{\mathbf{I}}$, for which the associated partition $\pi$ is non-crossing and strictly bm-ordered. However, this means that in the limit the summation can be reduced to sequences from $\operatorname{BMO}(\pi, \xi)$. Therefore, we have the following reduction.

Corollary 5.2. We have that

$$
\lim _{\xi \rightarrow \infty}\left(\varphi\left(\left(S_{\xi}\right)^{n}\right)-\sum_{k=1}^{n} \sum_{\pi \in \mathcal{N C}(n ; k)} \sum_{\mu \in \operatorname{BMO}(\pi, \xi)} C(\mu)\right)=0 .
$$

To prove (5.9) consider $\pi=\left(B_{1}, \ldots, B_{k}\right) \in \mathcal{N C}(n ; k)$ with the block cardinality $k_{j}:=\left|B_{j}\right|$ for $1 \leq j \leq k$. Let $\mu=\left(\mu_{1}, \ldots, \mu_{k}\right) \in \operatorname{BMO}(\pi, \xi)$, then $C(\mu)=$ $\prod_{j=1}^{k} \varphi\left(\left(X_{\mu_{j}}^{\xi}\right)^{k_{j}}\right)$ and hence, by the assumption (2) of Theorem 4.4, we have

$$
\lim _{\xi \rightarrow \infty} v(\xi)^{k} C(\mu)=\lambda^{k}
$$


In the next Lemma we prove that (5.9) can be viewed as passing from computing limit of moments (4.2) to combinatorial computation of approximate cardinalities (4.3). This is the point where we really need the uniform type of convergence in (4.1).

Lemma 5.3. If $\pi \in \mathcal{N C}(n ; k)$ and $\xi \in \mathbf{I}$, then, under the assumption (2) of Theorem 4.4 , we have

$$
\lim _{\xi \rightarrow \infty}\left(\sum_{\mu \in \operatorname{BMO}(\pi, \xi)} C(\mu)-\lambda^{k} \frac{|\operatorname{BMO}(\pi, \xi)|}{v(\xi)^{k}}\right)=0 .
$$

Proof: By the condition (4.1) of the uniform convergence, we have the following uniform estimate for $v(\xi)^{k} C(\mu)=\prod_{j=1}^{k} v(\xi) \varphi\left(\left(X_{\mu_{j}}^{\xi}\right)^{k_{j}}\right)$ : for each $\varepsilon>0$, there exists $\eta \in \Pi$ such that for all $\xi \succeq \eta$

$$
\sup _{\mu \in\left([0, \xi]_{\mathbf{I}}\right)^{k}}\left|v(\xi)^{k} C(\mu)-\lambda^{k}\right|<\varepsilon .
$$

Hence the difference

$$
\sum_{\mu \in \operatorname{BMO}(\pi, \xi)} C(\mu)-\lambda^{k} \frac{|\operatorname{BMO}(\pi, \xi)|}{v(\xi)^{k}}=\sum_{\mu \in \operatorname{BMO}(\pi, \xi)}\left(C(\mu)-\frac{\lambda^{k}}{v(\xi)^{k}}\right)
$$

can be estimated by

$$
\frac{1}{v(\xi)^{k}} \sum_{\mu \in \operatorname{BMO}(\pi, \xi)}\left|v(\xi)^{k} C(\mu)-\lambda^{k}\right|<\frac{|\operatorname{BMO}(\pi, \xi)|}{v(\xi)^{k}} \cdot \varepsilon \leq \varepsilon
$$

which gives the Lemma.

\section{Approximation of the cardinalities $|\operatorname{BMO}(\pi, \xi)|$.}

It follows from Corollary 5.2 and Lemma 5.3 that the limit in Theorem 4.4 can be equivalently computed as

$$
\lim _{\xi \rightarrow \infty} \varphi\left(\left(S_{\xi}\right)^{n}\right)=\sum_{k=1}^{n} \sum_{\pi \in \mathcal{N} \mathcal{C}(n ; k)} \lambda^{k} \lim _{\xi \rightarrow \infty} \frac{|\operatorname{BMO}(\pi, \xi)|}{v(\xi)^{k}} .
$$

Therefore, the proof of the main theorem has been reduced to properties of bmordered non-crossing partitions and positive symmetric cones. The following result is the main combinatorial property of the non-crossing partitions which are bmordered by elements of our positive cones.

Theorem 6.1. For $d \in \mathbb{N}$ let $\Pi=\Pi_{d}$ be one of the positive symmetric cones: $\Pi=\mathbb{R}_{+}^{d}, \Pi=\Lambda_{d}^{1}$ (the Lorentz light cones) or $\Pi=\operatorname{Symm}_{d}^{+}(\mathbb{R})$ (real symmetric positive definite matrices). Let $\pi \in \mathcal{N C}(n ; k)$ be a non-crossing partition of $[n]$ with $1 \leq k=b(\pi) \leq n$ blocks. Then the limits

$$
\begin{aligned}
\lim _{\xi \stackrel{\Pi}{\longrightarrow} \infty} \frac{|\operatorname{BMO}(\pi, \xi)|}{v(\xi)^{k}} & =V(\pi), \\
\lim _{\xi \rightarrow \infty} \varphi\left(\left(S_{\xi}\right)^{n}\right) & =\sum_{\pi \in \mathcal{N C}(n)} \lambda^{b(\pi)} V(\pi),
\end{aligned}
$$


exist. The function $V(\pi)$ depends on the positive cone $\Pi$ and its volume characteristic sequence $\left(\gamma_{n}\right)_{n \geq 1}$ and satisfies the recursion (4.4).

Proof: Observe that if we prove (6.2), then (6.3) will follow immediately from (6.1). The proof of (6.2) in Theorem 6.1 will be by the simultaneous induction on $n \in \mathbb{N}$ and on the number of blocks $1 \leq k \leq n$.

The case $1=k=n$ is trivial, since in this case $\pi=\{1\}$ is a singleton and thus $\operatorname{BMO}(\pi, \xi)=[0, \xi]_{\mathbf{I}}$. Hence the limit is of the form

$$
\lim _{\xi \stackrel{\Pi}{\longrightarrow} \infty} \frac{\left|[0, \xi]_{\mathbf{I}}\right|}{v(\xi)}=1=V(\pi) .
$$

Now, let us assume that a partition $\pi \in \mathcal{N C}(n ; k)$ is given with $n \geq 2$ and $1 \leq$ $k=b(\pi) \leq n$. Moreover, assume that for every $1 \leq k^{\prime}<k, 1 \leq n^{\prime}<n$ and every $\pi^{\prime} \in \mathcal{N} \mathcal{C}\left(n^{\prime}, k^{\prime}\right)$ the limit

$$
\lim _{\xi \Pi_{\infty}} \frac{\left|\operatorname{BMO}\left(\pi^{\prime}, \xi\right)\right|}{v(\xi)^{k^{\prime}}}=V\left(\pi^{\prime}\right)
$$

exists. We will construct an approximate recursive formula for the cardinalities $|\operatorname{BMO}(\pi, \xi)|$. For this purpose, we will use the notation introduced in Notation 4.3.

Thus, let $\pi=\pi_{1}^{\prime} \cup \pi_{2}^{\prime} \cup\left\{B_{1}\right\}$, where $1 \in B_{1}$. We choose the label $\mu_{1} \in[0, \xi]_{\mathbf{I}}$ for the block $B_{1}$. The labels for the blocks of $\pi_{2}^{\prime}$ can be chosen arbitrarily from $[0, \xi]_{\mathbf{I}}$ with the exception of the chosen $\mu_{1}$. However, this exception does not matter when we divide by $v(\xi)^{k}$ and let $\xi \stackrel{\Pi}{\rightarrow} \infty$. On the other hand, all the blocks of the subpartition $\pi_{1}^{\prime}$ are inside the block $B_{1}$, so the bm-order requires that the labels $\rho$ for the blocks of $\pi_{1}^{\prime}$ can be chosen only from $\left(\mu_{1}, \xi\right]_{\mathbf{I}}$, since they must satisfy $\mu_{1} \prec \rho \preceq \xi$. Moreover, they should be different from the (finite fixed number of) labels already chosen for the blocks of $\pi_{2}^{\prime}$. Again, dividing by $v(\xi)^{k}$ and taking the limit $\xi \stackrel{\Pi}{\rightarrow} \infty$ allows us to take, equivalently, all the possible labels $\mu_{1} \preceq \rho \preceq \xi$. However, by the change of variables $\rho \mapsto \rho-\mu_{1}$, we get an equivalent relation $0 \preceq \rho \preceq \xi-\mu_{1}$. This means that the number of bm-orders on $\pi_{1}^{\prime}$ with labels $\rho \in\left[\mu_{1}, \xi\right]_{\mathbf{I}}$ is the same as the number of bm-orders on $\pi_{1}^{\prime}$ with labels $\rho \in\left[0, \xi-\mu_{1}\right]_{\mathbf{I}}$. Therefore, we will obtain the following approximate recursive expression (writing $\mu$ instead of $\mu_{1}$ ):

$$
\frac{|\operatorname{BMO}(\pi, \xi)|}{v(\xi)^{k}} \approx \frac{1}{v(\xi)^{k}}\left(\sum_{\mu \in[0, \xi]_{\mathbf{I}}}\left|\operatorname{BMO}\left(\pi_{1}^{\prime}, \xi-\mu\right)\right|\right) \cdot\left|\operatorname{BMO}\left(\pi_{2}^{\prime}, \xi\right)\right|,
$$

meaning that the difference of both sides goes to zero as $\xi \stackrel{\Pi}{\rightarrow} \infty$. By the change of variables $\rho=\xi-\mu$ we can write this as

$$
\frac{|\operatorname{BMO}(\pi, \xi)|}{v(\xi)^{k}} \approx \frac{1}{v(\xi)^{k}}\left(\sum_{\rho \in[0, \xi]_{\mathbf{I}}}\left|\operatorname{BMO}\left(\pi_{1}^{\prime}, \rho\right)\right|\right) \cdot\left|\operatorname{BMO}\left(\pi_{2}^{\prime}, \xi\right)\right| .
$$

The right-hand side can be written as

$$
\frac{1}{v(\xi)}\left(\sum_{\rho \in[0, \xi]_{\mathbf{I}}} \frac{\left|\operatorname{BMO}\left(\pi_{1}^{\prime}, \rho\right)\right|}{v(\rho)^{k_{1}^{\prime}}} \cdot\left(\frac{v(\rho)}{v(\xi)}\right)^{k_{1}^{\prime}}\right) \cdot \frac{\left|\operatorname{BMO}\left(\pi_{2}^{\prime}, \xi\right)\right|}{v(\xi)^{k_{2}^{\prime}}},
$$

because $k=k_{1}^{\prime}+k_{2}^{\prime}+1$. Since $\pi_{j}^{\prime} \in \mathcal{N C}\left(n_{j}^{\prime}, k_{j}^{\prime}\right), 1 \leq k_{j}^{\prime} \leq k-1$ and $1 \leq n_{j}^{\prime} \leq n-1$ for $j=1,2$. Hence, by the induction assumption, we know that there exist the 
limits

$$
\lim _{\rho \rightarrow \infty} \frac{\left|\operatorname{BMO}\left(\pi_{1}^{\prime}, \rho\right)\right|}{v(\rho)^{k_{1}^{\prime}}}=V\left(\pi_{1}^{\prime}\right), \quad \lim _{\xi \rightarrow \infty} \frac{\left|\operatorname{BMO}\left(\pi_{2}^{\prime}, \xi\right)\right|}{v(\xi)^{k_{1}^{\prime}}}=V\left(\pi_{2}^{\prime}\right),
$$

which depend only on the partitions $\pi_{1}^{\prime}$ and $\pi_{2}^{\prime}$ and $V\left(\pi_{j}^{\prime}\right)(j=1,2)$ is just the notation for the limits. Hence, we get equivalently that

$$
\lim _{\xi \rightarrow \infty} \frac{|\operatorname{BMO}(\pi, \xi)|}{v(\xi)^{k}}=\lim _{\xi \rightarrow \infty} \frac{1}{v(\xi)}\left(\sum_{\rho \in[0, \xi]_{\mathbf{I}}} \frac{\left|\operatorname{BMO}\left(\pi_{1}^{\prime}, \rho\right)\right|}{v(\rho)^{k_{1}^{\prime}}} \cdot\left(\frac{v(\rho)}{v(\xi)}\right)^{k_{1}^{\prime}}\right) \cdot V\left(\pi_{2}^{\prime}\right)
$$

For proving Theorem 6.1 we will show that if $\lim _{\xi \rightarrow \infty} \frac{\left|\operatorname{BMO}\left(\pi_{1}^{\prime}, \xi\right)\right|}{v(\xi)^{k_{1}^{\prime}}}=V\left(\pi_{1}^{\prime}\right)$, then the limit

$$
\lim _{\xi \rightarrow \infty} \frac{1}{v(\xi)} \sum_{\rho \in[0, \xi]_{\mathbf{I}}} \frac{\left|\operatorname{BMO}\left(\pi_{1}^{\prime}, \rho\right)\right|}{v(\rho)^{k_{1}^{\prime}}} \cdot\left(\frac{v(\rho)}{v(\xi)}\right)^{k_{1}^{\prime}}=V\left(\pi_{1}^{\prime}\right) \cdot \gamma_{k_{1}^{\prime}+1}
$$

exists. Then it will follow that the limit $V(\pi):=\lim _{\xi \rightarrow \infty} \frac{|\operatorname{BMO}(\pi, \xi)|}{v(\xi)^{k}}$ exists and that the following recursive formula holds:

$$
V(\pi)=\gamma_{k_{1}^{\prime}+1} \cdot V\left(\pi_{1}^{\prime}\right) \cdot V\left(\pi_{2}^{\prime}\right)
$$

The proof of (6.10) will be carried out in the following Lemma.

Lemma 6.2. If $\pi \in \mathcal{N C}(n, k)$ is a non-crossing partition with $k=b(\pi)$ blocks, then for each of the positive symmetric cones $\Pi$ under consideration the limit

$$
\lim _{\xi \longrightarrow \infty} \frac{1}{v(\xi)} \sum_{\rho \in[0, \xi]_{\mathbf{I}}} \frac{|\operatorname{BMO}(\pi ; \rho)|}{v(\rho)^{k}} \cdot\left(\frac{v(\rho)}{v(\xi)}\right)^{k}=V(\pi) \cdot \gamma_{k+1},
$$

exists, where $\gamma_{k}=\gamma_{k}(\Pi)$ is the volume characteristic sequence of the cone $\Pi$ and the function $V=V_{\Pi}$ depends only on the cone $\Pi$ and the partition $\pi$.

Before starting the proof of the Lemma we explain its main idea, which is based on properties of the Riemann integral. The crucial technical property we will need is the following. For a partition $\pi \in \mathcal{N C}\left(n^{\prime}\right)$ (with $1 \leq n^{\prime} \leq n-1$ ), let $f_{\pi}: \Pi \rightarrow \mathbb{R}_{+}$be a continuous positive function on a positive cone $\Pi$ such that the limit $\lim f_{\pi}(\xi)=$ $\xi \stackrel{\Pi \rightarrow}{\rightarrow} \infty$

$V(\pi)$ exists. Let $\mathbf{I} \subset \Pi$ be the considered discrete subset (c.f. Definition 1.4). Then we claim that for any $k \in \mathbb{N}$ there exist (and are equal) the limits

$$
\lim _{\xi \rightarrow \infty} \frac{1}{v(\xi)} \sum_{\rho \in[0, \xi]_{\mathbf{I}}} f_{\pi}(\rho) \cdot\left(\frac{v(\rho)}{v(\xi)}\right)^{k}=\lim _{\xi \rightarrow \infty} \frac{1}{v(\xi)} \sum_{\rho \in[0, \xi]_{\mathbf{I}}} V(\pi) \cdot\left(\frac{v(\rho)}{v(\xi)}\right)^{k} .
$$

Both sides are the Riemann integral approximations, thus for the limit on the right-hand side we have

$$
\lim _{\xi \rightarrow \infty}\left|\frac{1}{v(\xi)} \sum_{\rho \in[0, \xi]_{\mathbf{I}}}\left(\frac{v(\rho)}{v(\xi)}\right)^{k}-\frac{1}{v(\xi)} \int_{\rho \in[0, \xi]}\left(\frac{v(\rho)}{v(\xi)}\right)^{k} d \rho\right|=0
$$

However, by Theorem 1.1 for any $\xi \in \Pi$

$$
\frac{1}{v(\xi)} \int_{\rho \in[0, \xi]}\left(\frac{v(\rho)}{v(\xi)}\right)^{k} d \rho=\frac{1}{v(\xi)^{k+1}} \int_{\rho \in[0, \xi]} v(\rho)^{k} d \rho=\gamma_{k+1}
$$


where $\gamma_{k}=\gamma_{k}(\Pi)$ is the volume characteristic sequence, which exists for the positive cone $\Pi$, as shown in Kula and Wysoczański (2010). Therefore, the right-hand side of (6.13) exists and equals $V(\pi) \gamma_{k+1}$.

Our proof that the limit on the left-hand side of (6.13) exists and is equal to the right-hand side will generalize the scheme given by the following property of positive continuous functions on the interval $[0,1]$.

Proposition 6.3. If $f \in C[0,1]$ is a non-negative continuous function and $\left(c_{n}\right)_{n \geq 1}$ is a convergent sequence with $\lim _{n} c_{n}=c$, then

$$
\lim _{n \rightarrow \infty} \frac{1}{n} \sum_{k=1}^{n} c_{k} \cdot f\left(\frac{k}{n}\right)=c \int_{0}^{1} f(x) d x
$$

To illustrate the idea we will use for general positive cones, we include the proof of this simple fact.

Proof: By the assumption, given $\varepsilon>0$, there exists $n_{0} \in \mathbb{N}$ such that for $n>n_{0}$ we have $\left|c_{n}-c\right|<\varepsilon$ and $\left|\frac{1}{n} \sum_{k=1}^{n} f\left(\frac{k}{n}\right)-\int_{0}^{1} f(x) d x\right|<\varepsilon$. Then, for such $n>n_{0}$, by splitting the summation we get the estimates

$$
\frac{1}{n}\left|\sum_{k=1}^{n}\left(c_{k}-c\right) \cdot f\left(\frac{k}{n}\right)\right| \leq \frac{1}{n} \sum_{k=1}^{n_{0}}\left|c_{k}-c\right| \cdot f\left(\frac{k}{n}\right)+\frac{1}{n} \sum_{k=n_{0}+1}^{n}\left|c_{k}-c\right| \cdot f\left(\frac{k}{n}\right)
$$

The first term on the right-hand side, as a finite sum with $n_{0}$ summands, tends to 0 as $n \rightarrow \infty$. The second term can be estimated by $\varepsilon \cdot \int_{0}^{1} f(x) d x$. This shows that the left-hand side tends to 0 , and thus, as a consequence, we get the conclusion of Proposition 6.3.

In our general case, where the positive reals are replaced by a positive symmetric cone, we have to consider a similar splitting of the summation $\frac{1}{v(\xi)} \sum_{\rho \in[0, \xi]_{I}} f_{\pi}(\rho)$. $\left(\frac{v(\rho)}{v(\xi)}\right)^{k}$, which depends on splitting the interval $[0, \xi]_{\mathbf{I}}$, given $\mu \in[0, \xi]_{\mathbf{I}}$ (the $\mu$ plays the role of $n_{0}$ ). However, in the partial ordered setting, the splitting is of the form

$$
[0, \xi]_{\mathbf{I}}=[0, \mu]_{\mathbf{I}} \cup(\mu, \xi]_{\mathbf{I}} \cup\left\{\rho \in[0, \xi]_{\mathbf{I}}: \rho \nsim \mu\right\} .
$$

The first two terms on the right-hand side play the same role as in the splitting $([0, n] \cap \mathbb{N})=\left(\left[0, n_{0}\right] \cap \mathbb{N}\right) \cup\left(\left(n_{0}, n\right] \cap \mathbb{N}\right)$, but the third term $\left\{\rho \in[0, \xi]_{\mathbf{I}}: \rho \nsim \mu\right\}$ introduces a new factor into play.

Now, for a given partition $\pi \in \mathcal{N C}(n ; k)$, we specify the continuous function $f_{\pi}: \Pi \rightarrow[0,1]$ by putting $f_{\pi}(\rho):=\frac{|\operatorname{BMO}(\pi ; \rho)|}{v(\rho)^{k}}, \rho \in \Pi$, for which we have formulated Lemma 6.2.

Proof of Lemma 6.2: We start the proof with considerations which are common for all three classes of the positive cones. For a non-crossing partition $\pi \in \mathcal{N C}(n, k)$, observe that because of the volume characteristic property (Theorem 1.1) it suffices 
to show that, approximately,

$$
\frac{1}{v(\xi)} \sum_{\rho \in[0, \xi]_{\mathbf{I}}} \frac{|\operatorname{BMO}(\pi ; \rho)|}{v(\rho)^{k}} \cdot\left(\frac{v(\rho)}{v(\xi)}\right)^{k} \approx V(\pi) \frac{1}{v(\xi)} \sum_{\rho \in[0, \xi]_{\mathbf{I}}}\left(\frac{v(\rho)}{v(\xi)}\right)^{k},
$$

since

$$
\gamma_{k+1}:=\frac{1}{v(\xi)} \int_{0}^{\xi}\left(\frac{v(\rho)}{v(\xi)}\right)^{k} d \rho \approx \frac{1}{v(\xi)} \sum_{\rho \in[0, \xi] \mathbf{I}}\left(\frac{v(\rho)}{v(\xi)}\right)^{k}
$$

Given $\varepsilon>0$, let $\mu \in \mathbf{I}$ be such that if $\rho \succeq \mu$, then $\left|\frac{|\operatorname{BMO}(\pi ; \rho)|}{v(\rho)^{k}}-V(\pi)\right|<\varepsilon$. Then, for such $\mu$ we will split the summation on the left-hand side of (6.12) into the sum of three terms

$$
\begin{gathered}
\frac{1}{v(\xi)} \sum_{\rho \in[0, \xi]_{\mathbf{I}}} \frac{|\operatorname{BMO}(\pi ; \rho)|}{v(\rho)^{k}}\left(\frac{v(\rho)}{v(\xi)}\right)^{k}=\frac{1}{v(\xi)} \sum_{\rho \in[\mu, \xi]_{\mathbf{I}}} \frac{|\operatorname{BMO}(\pi ; \rho)|}{v(\rho)^{k}}\left(\frac{v(\rho)}{v(\xi)}\right)^{k} \\
+\frac{1}{v(\xi)} \sum_{\rho \in[0, \mu]_{\mathbf{I}}} \frac{|\operatorname{BMO}(\pi ; \rho)|}{v(\rho)^{k}}\left(\frac{v(\rho)}{v(\xi)}\right)^{k}+\frac{1}{v(\xi)} \sum_{\mu \nsim \rho \in[0, \xi]_{\mathbf{I}}} \frac{|\operatorname{BMO}(\pi ; \rho)|}{v(\rho)^{k}}\left(\frac{v(\rho)}{v(\xi)}\right)^{k} .
\end{gathered}
$$

We first consider the second line, i.e. the two terms in (6.20). The first term is easy to estimate, since the sum is finite (as the set $[0, \mu]_{\mathbf{I}}$ is finite) and summands are bounded by 1 , so it can be estimated from above by $\frac{v(\mu)}{v(\xi)}$. Therefore it tends to zero, since $v(\xi) \rightarrow \infty$ as a consequence of the definition of $\xi \stackrel{\Pi}{\longrightarrow} \infty$. The next step of the proof will be to show that the second term in (6.20) tends to zero, and this will be done in Lemma 6.4 below separately for each case. Having done that, we will conclude that both sides of (6.19) are approximately equal, and thus we will be able to write

$$
\begin{aligned}
& \frac{1}{v(\xi)} \sum_{\rho \in[0, \xi]_{\mathbf{I}}}\left|\frac{|\operatorname{BMO}(\pi ; \rho)|}{v(\rho)^{k}}-V(\pi)\right|\left(\frac{v(\rho)}{v(\xi)}\right)^{k} \\
& \approx \frac{1}{v(\xi)} \sum_{\rho \in[\mu, \xi]_{\mathbf{I}}}\left|\frac{|\operatorname{BMO}(\pi ; \rho)|}{v(\rho)^{k}}-V(\pi)\right|\left(\frac{v(\rho)}{v(\xi)}\right)^{k} .
\end{aligned}
$$

Here the two sides differ only in the range of summation of the variable $\rho$. By the choice of $\mu$, the right-hand side of this expression can be estimated from above by

$$
\varepsilon \cdot \frac{1}{v(\xi)} \sum_{\rho \in[\mu, \xi]_{\mathbf{I}}}\left(\frac{v(\rho)}{v(\xi)}\right)^{k} \leq \varepsilon \cdot \frac{1}{v(\xi)} \sum_{\rho \in[0, \xi]_{\mathbf{I}}}\left(\frac{v(\rho)}{v(\xi)}\right)^{k} \rightarrow \varepsilon \cdot \gamma_{k+1},
$$

where the second approximation comes from the volume characteristic theorem. As a consequence, the left-hand side of (6.21) tends to zero, which is equivalent to the conclusion of Lemma 6.2.

Therefore in what follows we will show the following property.

Lemma 6.4. Given $\pi \in \mathcal{N C}(n ; k)$, suppose that for ever $\varepsilon>0$, there exists $\mu \in \mathbf{I}$ such that

$$
\left|\frac{|\operatorname{BMO}(\pi ; \rho)|}{v(\rho)^{k}}-V(\pi)\right|<\varepsilon \quad \text { if } \quad \rho \succeq \mu \text {. }
$$


Then, for each of the considered three classes of positive symmetric cones, we have

$$
\lim _{\xi \stackrel{\Pi}{\longrightarrow} \infty} \frac{1}{v(\xi)} \sum_{\mu \nsim \rho \in[0, \xi] \mathbf{I}} \frac{|\operatorname{BMO}(\pi ; \rho)|}{v(\rho)^{k}}\left(\frac{v(\rho)}{v(\xi)}\right)^{k}=0 .
$$

Proof of Lemma 6.4 for $\Pi=\mathbb{R}_{+}^{d}$. We will use the notation $\xi:=\left(a_{1} \leq \cdots \leq a_{d}\right)$, $\rho:=\left(b_{1} \leq \cdots \leq b_{d}\right), \mu:=\left(c_{1} \leq \cdots \leq c_{d}\right) \in \mathbf{I}:=\mathbb{N}^{d}, a:=v(\xi)=a_{1} \cdots a_{d}$ and, omitting the lim symbol, write (6.24) as

$$
\frac{1}{v(\xi)} \sum_{\mu \nsim \rho \in[0, \xi]_{\mathbf{I}}} \frac{|\operatorname{BMO}(\pi ; \rho)|}{v(\rho)^{k}}\left(\frac{v(\rho)}{v(\xi)}\right)^{k}=\frac{1}{a} \sum_{\mu \nsim \rho \in[0, \xi]_{\mathrm{I}}} \frac{|\operatorname{BMO}(\pi ; \rho)|}{v(\rho)^{k}} \prod_{j=1}^{d}\left(\frac{b_{j}}{a_{j}}\right)^{k} .
$$

The right-hand side is a sum of positive terms and to show that it tends to 0 we first write it as

$$
\frac{1}{a} \sum_{\mu \nsim \rho \in[0, \xi]_{\mathrm{I}}} \prod_{j=1}^{d}\left(\frac{b_{j}}{a_{j}}\right)^{k}=\prod_{j=1}^{d} \frac{1}{a_{j}} \sum_{b_{j}}\left(\frac{b_{j}}{a_{j}}\right)^{k} .
$$

We omit the range of summation for $b_{j}$, but it is understood that it is so that the condition $\mu \nsim \rho \in[0, \xi]_{\mathbf{I}}$ is satisfied. In these sums $b_{j} \leq a_{j}$ in general, so for every $1 \leq j \leq d$ we have $\frac{1}{a_{j}} \sum_{b_{j}}\left(\frac{b_{j}}{a_{j}}\right)^{k} \leq 1$. However, since $\rho \nsim \mu$, there is at least one index $1 \leq i \leq d$ such that $0 \leq b_{i} \leq c_{i}$. Then $\frac{b_{i}}{a_{i}} \leq \frac{c_{i}}{a_{i}} \leq \frac{c_{d}}{a_{1}}$, which is a uniform estimate independent of $\rho$. Hence, the right-hand side of (6.26) is estimated from above by $\left(\frac{c_{d}}{a_{1}}\right)^{k}$, which tends to 0 as $a_{1} \rightarrow \infty$. Therefore, the right-hand side of (6.26) tends to zero as $\xi \stackrel{\Pi}{\longrightarrow} \infty$. Thus the conclusion of Lemma 6.4 follows for $\Pi=\mathbb{R}_{+}^{d}$.

Proof of Lemma 6.4 for $\Pi=\Lambda_{d}^{1}$. The estimate

$$
0 \leq \frac{|\operatorname{BMO}(\pi ; \rho)|}{v_{d}(\rho)^{k}}\left(\frac{v_{d}(\rho)}{v_{d}(\xi)}\right)^{k} \leq 1
$$

gives that

$$
0 \leq \frac{1}{v_{d}(\xi)} \sum_{\mu \nsim \rho \in[0, \xi] \mathbf{I}} \frac{|\operatorname{BMO}(\pi ; \rho)|}{v_{d}(\rho)^{k}}\left(\frac{v_{d}(\rho)}{v_{d}(\xi)}\right)^{k} \leq \frac{|\Omega(\xi, \mu)|}{v_{d}(\xi)} .
$$

Therefore, it suffices to show that

$$
\lim _{\xi \rightarrow \infty} \frac{|\Omega(\xi, \mu)|}{v_{d}(\xi)}=0
$$

Given $\mu \in[0, \xi]_{\mathbf{I}}$, let $\Omega(\xi, \mu):=\left\{\rho \in[0, \xi]_{\mathbf{I}}: \rho \nsim \mu\right\}$ and observe that

$$
\Omega(\xi, \mu)=[0, \xi]_{\mathbf{I}} \backslash\left([\mu, \xi]_{\mathbf{I}} \cup[0, \mu]_{\mathbf{I}}\right),
$$

so that

$$
\frac{\Omega(\xi, \mu)}{v_{d}(\xi)} \approx 1-\frac{v_{d}(\xi-\mu)}{v_{d}(\xi)}-\frac{v_{d}(\mu)}{v_{d}(\xi)} .
$$

The last term on the right-hand side goes to zero since the numerator is fixed. Hence it suffices to prove that

$$
\lim _{\xi \rightarrow \infty} \frac{v_{d}(\xi-\mu)}{v_{d}(\xi)}=1
$$


With the notation $\xi:=(t ; x), \mu:=(s ; y) \in \mathbf{I}:=\mathbb{N} \times \mathbb{Z}^{d} \cap \Lambda_{d}^{1}$ we have that $v_{d}(\xi)=$ $c_{d}\left(t^{2}-\|x\|^{2}\right)^{\frac{d+1}{2}}$, hence we can write this as

$$
\lim _{\xi \rightarrow \infty}\left(\frac{(t-s)^{2}-\|x-y\|^{2}}{t^{2}-\|x\|^{2}}\right)^{\frac{d+1}{2}}=1,
$$

which is equivalent to

$$
\lim _{\xi \rightarrow \infty} \frac{(t-s)^{2}-\|x-y\|^{2}}{t^{2}-\|x\|^{2}}=1
$$

and to

$$
\lim _{\xi \rightarrow \infty} \frac{(t-s)^{2}-\|x-y\|^{2}-t^{2}+\|x\|^{2}}{t^{2}-\|x\|^{2}}=0 .
$$

Obviously (6.35) will follow if we prove that

$$
\lim _{\xi \rightarrow \infty} \frac{s^{2}-2 t s}{t^{2}-\|x\|^{2}}=0=\lim _{\xi \rightarrow \infty} \frac{\|x\|^{2}-\|x-y\|^{2}}{t^{2}-\|x\|^{2}} .
$$

Assume that $\lim _{\xi \rightarrow \infty}(t-\|x\|)=+\infty$, then also $\lim _{\xi \rightarrow \infty}(t+\|x\|)=+\infty$. For proving (6.36) we write

$$
\begin{aligned}
\frac{s^{2}-2 t s}{t^{2}-\|x\|^{2}} & =\frac{s^{2}-2 t s}{t+\|x\|} \cdot \frac{1}{t-\|x\|}, \\
\frac{\|x\|^{2}-\|x-y\|^{2}}{t^{2}-\|x\|^{2}} & =\frac{\|x\|^{2}-\|x-y\|^{2}}{t+\|x\|} \cdot \frac{1}{t-\|x\|},
\end{aligned}
$$

and observe that the first terms on the right-hand side are bounded, while the second tend to zero.

Proof of Lemma 6.4 for $\Pi=\operatorname{Symm}_{d}^{+}(\mathbb{R})$. It is known (see Horn and Johnson, 1985, Corollary 7.7.4) that the partial order $\rho \preceq \xi$ for positive definite real symmetric matrices $\xi, \rho \in \operatorname{Symm}_{d}^{+}(\mathbb{R})$ implies that their ordered eigenvalues can be compared. On the other hand, the product of the eigenvalues (i.e. the determinant) of $\xi \in$ $\operatorname{Symm}_{d}^{+}(\mathbb{R})$ gives the volume of the interval $[0, \xi]$. However, for our proof we only need the following simple property of the partial order, which is well known, and also easily follows from the above-mentioned Corollary in Horn and Johnson (1985). Namely, if $\xi \in \Pi$ has ordered eigenvalues $0 \leq \xi_{1} \leq \cdots \leq \xi_{d}$, then $a \cdot \mathbb{I} \preceq_{\Pi} \xi \preceq_{\Pi} b \cdot \mathbb{I}$ for any $a \leq \xi_{1} \leq \xi_{d} \leq b$ where $\mathbb{I}$ is the identity $d \times d$ matrix.

For proving Lemma 6.4 in this case assume that $\mu \in \Pi$ is so that for $\mu \preceq \rho$ we have $\left|\frac{\operatorname{BMO}(\pi ; \rho)}{v(\rho)^{k}}-V(\pi)\right|<\varepsilon$. Let $a=\|\mu\|$ be the largest eigenvalue (operator norm) of the positive definite matrix $\mu$. Then $\mu \preceq a \mathbb{I}$, so that for $a \mathbb{I} \preceq \rho$ the condition $\left|\frac{\operatorname{BMO}(\pi ; \rho)}{v(\rho)^{k}}-V(\pi)\right|<\varepsilon$ is also satisfied. As before, for proving Lemma 6.4 it suffices to show that

$$
\lim _{\xi \stackrel{\Pi}{\longrightarrow} \infty} \frac{1}{v(\xi)} \sum_{\mu \nsim \rho \in[0, \xi]_{\mathbf{I}}} \frac{|\mathrm{BMO}(\pi ; \rho)|}{v(\rho)^{k}}\left(\frac{v(\rho)}{v(\xi)}\right)^{k}=0 .
$$

Recall that each term in the summation is less than or equal 1. Since $\rho \nsim \mu$ implies that either $\rho \prec a \mathbb{I}$ or $\rho \nsim a \mathbb{I}$, the summation above will be estimated from above 
by the summation over $\rho \in[0, \xi]_{\mathbf{I}}$ where either $0 \preceq \rho \preceq a \mathbb{I}$ or $\rho \nsim a \mathbb{I}$. However, for $0 \preceq \rho \preceq a \mathbb{I}$ the sum is finite, hence

$$
\lim _{\xi \stackrel{\Pi}{\longrightarrow} \infty} \frac{1}{v(\xi)} \sum_{a \mathbb{I} \succ \rho \in[0, \xi]_{\mathbf{I}}} \frac{|\operatorname{BMO}(\pi ; \rho)|}{v(\rho)^{k}}\left(\frac{v(\rho)}{v(\xi)}\right)^{k}=0 .
$$

Therefore, we only need to show that

$$
\lim _{\xi \stackrel{\Pi}{\longrightarrow} \infty} \frac{1}{v(\xi)} \sum_{a \mathbb{I} \infty \rho \in[0, \xi]_{\mathbf{I}}} \frac{|\operatorname{BMO}(\pi ; \rho)|}{v(\rho)^{k}}\left(\frac{v(\rho)}{v(\xi)}\right)^{k}=0 .
$$

Let $0 \leq \rho_{1} \leq \cdots \leq \rho_{d}$ be the eigenvalues of a given $\rho$ with $a \mathbb{I} \nsim \rho \in[0, \xi]_{\mathbf{I}}$. Then it cannot be $\rho_{j} \geq a$ for all $1 \leq j \leq d$, since then it would be $\rho \succ a \mathbb{I}$. Hence, for each such $\rho$ there exists $1 \leq i \leq d$ such that $\rho_{i}<a$, and, if $0<\xi_{1} \leq \cdots \leq \xi_{d}$ are the eigenvalues of $\xi$, then this implies the estimate $0<\frac{\rho_{i}}{\xi_{i}}<\frac{a}{\xi_{i}} \leq \frac{a}{\xi_{1}}$, which is uniform for all such $\rho$. Moreover, since $\frac{v(\rho)}{v(\xi)}=\left(\prod_{j=1}^{d} \frac{\rho_{j}}{\xi_{j}}\right)^{s}$ and $\frac{\rho_{j}}{\xi_{j}} \leq 1$ for $1 \leq j \leq d$, the summation in (6.39) is estimated from above by $\left(\frac{a}{\xi_{1}}\right)^{s k}$, which tends to 0 as $\xi \stackrel{\Pi}{\longrightarrow} \infty$. This proves Lemma 6.4 for the positive symmetric cone $\operatorname{Symm}_{d}^{+}(\mathbb{R})$.

In this fashion, we have also proved Lemma 6.2.

Since the Lemma was the only remaining ingredient for proving Theorem 6.1, we conclude that this theorem holds.

\section{Final remarks}

The results of this paper can be easily extended to two classes of non-symmetric cones, studied in Oussi and Wysoczański (2019), for which the volume characteristic was determined. In particular, for the sectorial cones $\Omega_{\mathbf{u}}^{n}:=\left\{\sum_{j=1}^{n} a_{j} u_{j}\right.$ : $\left.a_{1}, \ldots, a_{n} \geq 0\right\}$, defined by a collection $\mathbf{u}:=\left(u_{1}, \ldots, u_{n}\right)$ of linearly independent vectors $u_{1}, \ldots, u_{n} \in R^{n}$, whose determinant $D=\operatorname{det}(\mathbf{u})=\operatorname{det}\left(u_{1}, \ldots, u_{n}\right)$ is positive, one gets $\gamma_{k}\left(\Omega_{\mathbf{u}}^{n}\right)=\frac{1}{D \cdot k^{n}}$. The same can be done for the circular cones $C_{\theta}^{n}:=\left\{(t ; x) \in \mathbb{R}_{+} \times \mathbb{R}^{n}:\|x\| \leq t \cdot \tan \theta\right\}$, for which the volume characteristic sequence is the same as for the Lorentz cones $\Lambda_{1}^{n}$.

It is still to be investigated the volume characteristic property for the remaining positive symmetric cones (according to the classification in Faraut and Korányi, 1994), namely for the hermitian positive definite $n \times n$ matrices with complex entries or with quaternionic entries $(n \in \mathbb{N})$, and the exceptional $3 \times 3$ matrices with octonion entries. This would be the necessary ingredient in our method of proving the bm-LSN for these positive cones.

Here we list some open problems:

(1) determine the bm-LSN limit measures for every positive cone in question,

(2) find the volume characteristic for any positive symmetric cone,

(3) extend bm-LSN to operator-valued random variables,

(4) find bm-convolution of measures and prove related bm-LSN,

(5) find operator models for bm-LSN for positive symmetric cones. 


\section{Acknowledgements}

We would like to thank Eric Ricard, Franz Lehner and Romuald Lenczewski for their helpful comments. The paper has been significantly improved due to remarks and comments of the referee. In particular the formulations of Remark 2.3 and Theorem 3.12 and the construction of the partition $\sigma$ in Lemma 3.11, as well as the idea of using more combinatorial proofs, are due to his suggestion.

\section{References}

Anshelevich, M. Free stochastic measures via noncrossing partitions. Adv. Math., 155 (1), 154-179 (2000). MR1789851.

Anshelevich, M. and Williams, J. D. Operator-valued monotone convolution semigroups and an extension of the Bercovici-Pata bijection. Doc. Math., 21, 841-871 (2016). MR3548135.

Arizmendi, O. and Hasebe, T. On a class of explicit Cauchy-Stieltjes transforms related to monotone stable and free Poisson laws. Bernoulli, 19 (5B), 2750-2767 (2013). MR3160570.

Avitzour, D. Free products of $C^{*}$-algebras. Trans. Amer. Math. Soc., 271 (2), 423-435 (1982). MR654842.

Belavkin, V. P. A reconstruction theorem for a quantum random field. Uspekhi Mat. Nauk, 39 (2(236)), 137-138 (1984). MR740006.

Belavkin, V. P. A reconstruction theorem for a quantum random process. Teoret. Mat. Fiz., 62 (3), 408-431 (1985). MR791208.

Belinschi, S. T., Popa, M., and Vinnikov, V. On the operator-valued analogues of the semicircle, arcsine and Bernoulli laws. J. Operator Theory, 70 (1), 239-258 (2013). MR3085826.

Ben Ghorbal, A. and Schürmann, M. Non-commutative notions of stochastic independence. Math. Proc. Cambridge Philos. Soc., 133 (3), 531-561 (2002). MR1919720.

Ben Ghorbal, A. and Schürmann, M. Quantum stochastic calculus on Boolean Fock space. Infin. Dimens. Anal. Quantum Probab. Relat. Top., 7 (4), 631-650 (2004). MR2105916.

Bożejko, M. On $\Lambda(p)$ sets with minimal constant in discrete noncommutative groups. Proc. Amer. Math. Soc., 51, 407-412 (1975). MR390658.

Bożejko, M. Positive definite functions on the free group and the noncommutative Riesz product. Boll. Un. Mat. Ital. A (6), 5 (1), 13-21 (1986). MR833375.

Bożejko, M. Uniformly bounded representations of free groups. J. Reine Angew. Math., 377, 170-186 (1987). MR887407.

Bożejko, M. Deformed Fock spaces, Hecke operators and monotone Fock space of Muraki. Demonstratio Math., 45 (2), 399-413 (2012). MR2963077.

Bożejko, M., da Silva, J. L., Kuna, T., and Lytvynov, E. Approximation of a free Poisson process by systems of freely independent particles. Infin. Dimens. Anal. Quantum Probab. Relat. Top., 21 (3), 1850020, 25 (2018). MR3860253.

Bożejko, M., Krystek, A. D., and Wojakowski, L. u. J. Remarks on the $r$ and $\Delta$ convolutions. Math. Z., 253 (1), 177-196 (2006). MR2206642.

Bożejko, M., Kümmerer, B., and Speicher, R. q-Gaussian processes: noncommutative and classical aspects. Comm. Math. Phys., 185 (1), 129-154 (1997). MR1463036. 
Bożejko, M., Leinert, M., and Speicher, R. Convolution and limit theorems for conditionally free random variables. Pacific J. Math., 175 (2), 357-388 (1996). MR1432836.

Bożejko, M. and Wysoczański, J. New examples of convolutions and noncommutative central limit theorems. In Quantum probability (Gdańsk, 1997), volume 43 of Banach Center Publ., pp. 95-103. Polish Acad. Sci. Inst. Math., Warsaw (1998). MR1649712.

Bożejko, M. and Wysoczański, J. Remarks on $t$-transformations of measures and convolutions. Ann. Inst. H. Poincaré Probab. Statist., 37 (6), 737-761 (2001). MR1863276.

Buchholz, A. Norm of convolution by operator-valued functions on free groups. Proc. Amer. Math. Soc., 127 (6), 1671-1682 (1999). MR1476122.

Faraut, J. and Korányi, A. Analysis on symmetric cones. Oxford Mathematical Monographs. The Clarendon Press, Oxford University Press, New York (1994). ISBN 0-19-853477-9. MR1446489.

Franz, U. Unification of Boolean, monotone, anti-monotone, and tensor independence and Lévy processes. Math. Z., 243 (4), 779-816 (2003). MR1974583.

Hasebe, T. Conditionally monotone independence I: Independence, additive convolutions and related convolutions. Infin. Dimens. Anal. Quantum Probab. Relat. Top., 14 (3), 465-516 (2011a). MR2847249.

Hasebe, T. Independence generalizing monotone and Boolean independences. In Quantum probability and related topics, volume 27 of $Q P-P Q$ : Quantum Probab. White Noise Anal., pp. 190-201. World Sci. Publ., Hackensack, NJ (2011b). MR2799124.

Hasebe, T. and Saigo, H. The monotone cumulants. Ann. Inst. Henri Poincaré Probab. Stat., 47 (4), 1160-1170 (2011). MR2884229.

Hasebe, T. and Saigo, H. On operator-valued monotone independence. Nagoya Math. J., 215, 151-167 (2014). MR3263527.

Hiai, F. and Petz, D. The semicircle law, free random variables and entropy, volume 77 of Mathematical Surveys and Monographs. American Mathematical Society, Providence, RI (2000). ISBN 0-8218-2081-8. MR1746976.

Hora, A. and Obata, N. Quantum probability and spectral analysis of graphs. Theoretical and Mathematical Physics. Springer, Berlin (2007). ISBN 978-3-54048862-0. MR2316893.

Horn, R. A. and Johnson, C. R. Matrix analysis. Cambridge University Press, Cambridge (1985). ISBN 0-521-30586-1. MR832183.

Jekel, D. and Liu, W. An operad of non-commutative independences defined by trees. Dissertationes Math., 553, 100 (2020). MR4159542.

Kula, A. and Wysoczański, J. Noncommutative Brownian motions indexed by partially ordered sets. Infin. Dimens. Anal. Quantum Probab. Relat. Top., 13 (4), 629-661 (2010). MR2754321.

Kula, A. and Wysoczański, J. An example of a Boolean-free type central limit theorem. Probab. Math. Statist., 33 (2), 341-352 (2013). MR3158560.

Lenczewski, R. Unification of independence in quantum probability. Infin. Dimens. Anal. Quantum Probab. Relat. Top., 1 (3), 383-405 (1998). MR1638097.

Lenczewski, R. Decompositions of the free additive convolution. J. Funct. Anal., 246 (2), 330-365 (2007). MR2321046. 
Lenczewski, R. and Sałapata, R. Discrete interpolation between monotone probability and free probability. Infin. Dimens. Anal. Quantum Probab. Relat. Top., 9 (1), 77-106 (2006). MR2214503.

Liu, W. Extended de Finetti theorems for boolean independence and monotone independence. Trans. Amer. Math. Soc., 370 (3), 1959-2003 (2018). MR3739198.

Lu, Y. G. An interacting free Fock space and the arcsine law. Probab. Math. Statist., 17 (1, Acta Univ. Wratislav. No. 1928), 149-166 (1997). MR1455615.

Młotkowski, W. $\Lambda$-free probability. Infin. Dimens. Anal. Quantum Probab. Relat. Top., 7 (1), 27-41 (2004a). MR2044286.

Młotkowski, W. Limit theorems in $\Lambda$-Boolean probability. Infin. Dimens. Anal. Quantum Probab. Relat. Top., 7 (3), 449-459 (2004b). MR2085643.

Muraki, N. Monotonic independence, monotonic central limit theorem and monotonic law of small numbers. Infin. Dimens. Anal. Quantum Probab. Relat. Top., 4 (1), 39-58 (2001). MR1824472.

Muraki, N. The five independences as quasi-universal products. Infin. Dimens. Anal. Quantum Probab. Relat. Top., 5 (1), 113-134 (2002). MR1895232.

Nica, A. and Speicher, R. Lectures on the combinatorics of free probability, volume 335 of London Mathematical Society Lecture Note Series. Cambridge University Press, Cambridge (2006). ISBN 978-0-521-85852-6; 0-521-85852-6. MR2266879.

Oussi, L. and Wysoczański, J. bm-central limit theorems associated with nonsymmetric positive cones. Probab. Math. Statist., 39 (1), 183-197 (2019). MR3964391.

Popa, M. A combinatorial approach to monotonic independence over a $C^{*}$-algebra. Pacific J. Math., 237 (2), 299-325 (2008). MR2421124.

Proskurin, D. P. and Iksanov, A. M. The interpolation between the classical and monotone independence given by the twisted CCR. Opuscula Math., (23), 63-69 (2003). MR2061458.

Pusz, W. and Woronowicz, S. L. Twisted second quantization. Rep. Math. Phys., 27 (2), 231-257 (1989). MR1067498.

Speicher, R. A new example of "independence" and "white noise". Probab. Theory Related Fields, 84 (2), 141-159 (1990). MR1030725.

Speicher, R. Free probability theory and non-crossing partitions. Sém. Lothar. Combin., 39, Art. B39c, 38 pp. (1997a). MR1490288.

Speicher, R. On universal products. In Free probability theory (Waterloo, ON, 1995), volume 12 of Fields Inst. Commun., pp. 257-266. Amer. Math. Soc., Providence, RI (1997b). MR1426844.

Speicher, R. Combinatorial theory of the free product with amalgamation and operator-valued free probability theory. Mem. Amer. Math. Soc., 132 (627), $\mathrm{x}+88$ (1998). MR1407898.

Speicher, R. and Woroudi, R. Boolean convolution. In Free probability theory (Waterloo, ON, 1995), volume 12 of Fields Inst. Commun., pp. 267-279. Amer. Math. Soc., Providence, RI (1997). MR1426845.

Speicher, R. and Wysoczański, J. Mixtures of classical and free independence. Arch. Math. (Basel), 107 (4), 445-453 (2016). MR3552222.

Voiculescu, D. Symmetries of some reduced free product $C^{*}$-algebras. In Operator algebras and their connections with topology and ergodic theory (Buşteni, 1983), volume 1132 of Lecture Notes in Math., pp. 556-588. Springer, Berlin (1985). MR799593. 
Voiculescu, D. Addition of certain noncommuting random variables. J. Funct. Anal., 66 (3), 323-346 (1986). MR839105.

Voiculescu, D. Free probability theory: random matrices and von Neumann algebras. In Proceedings of the International Congress of Mathematicians, Vol. 1, 2 (Zürich, 1994), pp. 227-241. Birkhäuser, Basel (1995). MR1403924.

Voiculescu, D. V., Dykema, K. J., and Nica, A. Free random variables. A noncommutative probability approach to free products with applications to random matrices, operator algebras and harmonic analysis on free groups, volume 1 of CRM Monograph Series. American Mathematical Society, Providence, RI (1992). ISBN 0-8218-6999-X. MR1217253.

von Waldenfels, W. An algebraic central limit theorem in the anti-commuting case. Z. Wahrscheinlichkeitstheorie und Verw. Gebiete, 42 (2), 135-140 (1978). MR467881.

Wojakowski, Ł. Probability interpolating between free and Boolean. Dissertationes Math., 446, 45 (2007). MR2308717.

Wysoczański, J. Monotonic independence on the weakly monotone Fock space and related Poisson type theorem. Infin. Dimens. Anal. Quantum Probab. Relat. Top., 8 (2), 259-275 (2005). MR2146316.

Wysoczański, J. Monotonic independence associated with partially ordered sets. Infin. Dimens. Anal. Quantum Probab. Relat. Top., 10 (1), 17-41 (2007). MR2310562.

Wysoczański, J. bm-central limit theorems for positive definite real symmetric matrices. Infin. Dimens. Anal. Quantum Probab. Relat. Top., 11 (1), 33-51 (2008). MR2401871.

Wysoczański, J. bm-independence and bm-central limit theorems associated with symmetric cones. Infin. Dimens. Anal. Quantum Probab. Relat. Top., 13 (3), 461-488 (2010). MR2729595. 